\title{
The Second Noether Theorem on Time Scales
}

\author{
Agnieszka B. Malinowska ${ }^{1}$ and Natália Martins ${ }^{2}$ \\ ${ }^{1}$ Faculty of Computer Science, Bialystok University of Technology, 15-351 Bialystok, Poland \\ ${ }^{2}$ Center for Research and Development in Mathematics and Applications (CIDMA), Department of Mathematics, \\ University of Aveiro, 3810-193 Aveiro, Portugal \\ Correspondence should be addressed to Agnieszka B. Malinowska; a.malinowska@pb.edu.pl
}

Received 23 August 2013; Accepted 9 October 2013

Academic Editor: Delfim F. M. Torres

Copyright (C) 2013 A. B. Malinowska and N. Martins. This is an open access article distributed under the Creative Commons Attribution License, which permits unrestricted use, distribution, and reproduction in any medium, provided the original work is properly cited.

We extend the second Noether theorem to variational problems on time scales. As corollaries we obtain the classical second Noether theorem, the second Noether theorem for the $h$-calculus and the second Noether theorem for the $q$-calculus.

\section{Introduction}

In 1915, general relativity was almost a finished theory, but still there was a problem regarding the conservation of energy. David Hilbert asked for help Emmy Noether. She solved the problem proving two remarkable theorems that relate to the invariance of a variational integral with properties of its Euler-Lagrange equations. These results were published in 1918 in the paper Invariante Variations probleme [1]. Noether was described by many important scientists, such as Pavel Alexandrov, Albert Einstein, Jean Dieudonné, and David Hilbert, as the most important woman in the history of mathematics. In order to get a good exposition of the history of Emmy Noether and her important contributions to fundamental physics and mathematics, we refer the reader to the recent book [2]. This book explains very clearly that it took too much time before mathematicians and physicists began to recognize the importance of Noether's theorems: until 1950 Noether's theorems were poorly understood and Noether's name disappeared almost entirely.

The first theorem in [1], usually known as Noether's theorem, guarantees that the invariance of a variational integral with respect to continuous symmetry transformations that depend on $\rho$ parameters implies the existence of $\rho$ conserved quantities along the Euler-Lagrange extremals. Such transformations are global transformations. Noether's theorem explains all conservation laws of mechanics: conservation of energy comes from invariance of the system under time translations; conservation of linear momentum comes from invariance of the system under spacial translations; conservation of angular momentum reflects invariance with respect to spatial rotations.

The first Noether theorem is nowadays a well-known tool in modern theoretical physics, engineering, and the calculus of variations [3-9]. Inexplicably, it is still not well known that the famous paper of Emmy Noether includes another important result, the second Noether theorem, which applies to variational problems that are invariant under a certain group of transformations, a so-called infinite continuous group, which depends on arbitrary functions and their derivatives (see also [10]). Such transformations are local transformations because they can affect every part of the system differently. Noether's second theorem states that if a variational integral has an infinite-dimensional Lie algebra of infinitesimal symmetries parameterized linearly by $r$ arbitrary functions and their derivatives up to a given order $m$, then there are $r$ identities between Euler-Lagrange expressions and their derivatives up to order $m$. These identities Noether called them "dependencies." For example, the Bianchi identities, in the general theory of relativity, are examples of such "dependencies." Noether's second theorem has applications in general relativity, electrodynamics, hydromechanics, quantum chromodynamics, and other gauge field theories. Motivated by the important applications of the second Noether theorem, our goal in this paper is to generalize this result proving that the second Noether 
theorem is valid for an infinite number of time scales. As we will see, in the particular case where the time scale $\mathbb{T}$ is $\mathbb{R}$, we get from our result the classical second Noether theorem; when $\mathbb{T}=\mathbb{Z}$, we obtain the analogue of the second Noether theorem for the difference calculus of variations; when $\mathbb{T}=q^{\mathbb{N}_{0}}$ (for some $q>1$ ), we obtain a new result: the second Noether theorem for the $q$-calculus (quantum calculus). For more on the theory of quantum calculus and quantum calculus of variation, we refer to [11-21].

The theory of time scales was introduced in 1988 by Hilger in his Ph.D. thesis [22] as a means of unifying theories of differential calculus and difference calculus into a single theory. With a short time this unification aspect has been supplemented by the extension and generalization features. The time scale calculus allows to consider more complex time domains, such as $h \mathbb{Z}, \mathbb{T}=q^{\mathbb{N}_{0}}$, or hybrid domains. The study of the calculus of variations in the context of time scales had its beginning only in 2004 with the paper [23] of Bohner (see also [24]). Since then, the variational calculus on time scales advanced fairly quickly, as can be verified with the large number of published papers on the subject [25-35]. Noether's first theorem has been extended to the variational calculus on time scales using several approaches $[4,9,36]$, while the second Noether theorem on times scales is still not available in the literature. So, there is evidently a need for a time scale analogue of Noether's second theorem.

The paper is organized as follows. In Section 2, we review some preliminaries about single-variable variational calculus on time scales; for example, we recall the EulerLagrange equation for a delta variational problem. Our main results are stated in Section 3. Namely, in Subsection 3.1, we prove Noether's second theorem for variational problems involving a single delta integral (with and without transformation of time) and in Section 3.2 we prove Noether's second theorem for variational problems involving multiple delta integrals (without transformation of time). Section 4 provides a concrete example of application of our results. Finally, in Section 5, we present some concluding remarks.

\section{Preliminaries}

In this paper, we assume the reader to be familiar with the calculus on time scales. For a good introduction to the theory of time scales, we refer to the well-known books in this field $[37,38]$. The first developments on time scale calculus were done essentially using the delta-calculus. However, for some applications, in particular to solve problems of the calculus of variations and control theory in economics, it is often more convenient to work backwards in time, that is, using the nabla-calculus. In this paper, we are concerned with the delta-calculus. It is clear that all the arguments used in the proofs of our results can be modified to work for the nablacalculus. In what follows, we review some preliminaries about the variational calculus on time scales needed in this paper.

Let $\mathbb{T}$ be a given time scale, $n \in \mathbb{N}$, and let $L: \mathbb{T} \times$ $\mathbb{R}^{n} \times \mathbb{R}^{n} \rightarrow \mathbb{R}$ be continuous, together with its partial delta derivatives of first and second order with respect to $t$ and partial usual derivatives of the first and second order with respect to the other variables. Suppose that $a, b \in \mathbb{T}$, and $a<b$. We consider the following optimization problem on T:

$$
\mathscr{L}[y]=\int_{a}^{b} L\left(t, y^{\sigma}(t), y^{\Delta}(t)\right) \Delta t \longrightarrow \text { extremize }
$$

where the set of admissible functions are

$$
\begin{gathered}
\mathscr{D}=\left\{y \mid y:[a, b] \cap \mathbb{T} \longrightarrow \mathbb{R}^{n}, y \in C_{\mathrm{rd}}^{1}\left([a, b] \cap \mathbb{T}, \mathbb{R}^{n}\right),\right. \\
y(a)=\alpha, y(b)=\beta\},
\end{gathered}
$$

for some $\alpha, \beta \in \mathbb{R}^{n}$, and where $\sigma$ is the forward jump operator, $y^{\Delta}$ is the delta-derivative of $y$, and, for $i \in \mathbb{N}$,

$$
\begin{aligned}
C_{\mathrm{rd}}^{i}\left([a, b] \cap \mathbb{T}, \mathbb{R}^{n}\right):=\{ & y \mid y:[a, b] \cap \mathbb{T} \longrightarrow \mathbb{R}^{n}, \\
& \left.y^{\Delta^{i}} \text { is rd-continuous on }[a, b]^{k^{i}}\right\} .
\end{aligned}
$$

As usual, $y^{\sigma}(t)$ denotes $y(\sigma(t))$ and $[a, b]^{\kappa^{i}}:=\left[a, \rho^{i}(b)\right]$, where $\rho$ is the backward jump operator. By extremize, we mean maximize or minimize.

In what follows, all intervals are time scales intervals; that is, we simply write $[a, b]$ to denote the set $[a, b] \cap \mathbb{T}$. Let $y:=$ $\left(y_{1}, \ldots, y_{n}\right)$ and denote by $\partial L / \partial y_{k}$ the partial derivative of $L$ with respect to $y_{k}$.

Definition 1. We say that $y_{*} \in C_{\mathrm{rd}}^{1}\left([a, b], \mathbb{R}^{n}\right)$ is a local minimizer (resp., local maximizer) for problem (1) if there exists $\delta>0$ such that

$$
\mathscr{L}\left[y_{*}\right] \leq \mathscr{L}[y] \quad\left(\text { resp., } \mathscr{L}\left[y_{*}\right] \geq \mathscr{L}[y]\right),
$$

for all $y \in C_{\mathrm{rd}}^{1}\left([a, b], \mathbb{R}^{n}\right)$ satisfying the boundary conditions $y(a)=\alpha, y(b)=\beta$, and

$$
\begin{aligned}
\left\|y-y_{*}\right\|:= & \sup _{t \in[a, b]^{\kappa}}\left|y^{\sigma}(t)-y_{*}^{\sigma}(t)\right| \\
& +\sup _{t \in[a, b]^{\kappa}}\left|y^{\Delta}(t)-y_{*}^{\Delta}(t)\right|<\delta,
\end{aligned}
$$

where $|\cdot|$ denotes a norm in $\mathbb{R}^{n}$.

Definition 2. We say that $\eta \in C_{\mathrm{rd}}^{1}\left([a, b], \mathbb{R}^{n}\right)$ is an admissible variation for problem (1) provided that $\eta(a)=\eta(b)=0$.

Definition 3. A function $f:[a, b] \times \mathbb{R} \rightarrow \mathbb{R}$ is called continuous in the second variable, uniformly in the first variable, if for each $\epsilon>0$, there exists $\delta>0$ such that $\left|x_{1}-x_{2}\right|<\delta$ implies $\left|f\left(t, x_{1}\right)-f\left(t, x_{2}\right)\right|<\epsilon$ for all $t \in[a, b]$.

Lemma 4 (see [23]). Suppose that $\eta:=\left(\eta_{1}, \ldots, \eta_{n}\right)$ is an admissible variation for problem $(1)$ and $y:=\left(y_{1}, \ldots, y_{n}\right) \in \mathscr{D}$. Let $\phi: \mathbb{R} \rightarrow \mathbb{R}$ and $f:[a, b] \times \mathbb{R} \rightarrow \mathbb{R}$ be defined, respectively, 
by $\phi(\epsilon):=\mathscr{L}[y+\epsilon \eta]$ and $f(t, \epsilon):=L\left(t, y^{\sigma}(t)+\epsilon \eta^{\sigma}(t), y^{\Delta}(t)+\right.$ $\left.\epsilon \eta^{\Delta}(t)\right)$. If $\partial f / \partial \epsilon$ is continuous in $\epsilon$, uniformly in $t$, then

$$
\begin{aligned}
\dot{\phi}(0)=\int_{a}^{b} \sum_{k=1}^{n}\left(\frac{\partial L}{\partial y_{k}^{\sigma}}\left(t, y^{\sigma}(t), y^{\Delta}(t)\right) \eta_{k}^{\sigma}(t)\right. \\
\left.+\frac{\partial L}{\partial y_{k}^{\Delta}}\left(t, y^{\sigma}(t), y^{\Delta}(t)\right) \eta_{k}^{\Delta}(t)\right) \Delta t .
\end{aligned}
$$

Next, we present the following result that is a fundamental tool in the calculus of variations on time scales.

Theorem 5 (Euler-Lagrange equation on time scales [23]). If $y_{*}$ is a weak local extremizer for problem (1) and L satisfies the assumption of Lemma 4, for every $y$ and $\eta$, then the components of $y_{*}$ satisfy the $n$ Euler-Lagrange equations:

$$
\begin{array}{r}
\frac{\Delta}{\Delta t} \frac{\partial L}{\partial y_{k}^{\Delta}}\left(t, y^{\sigma}(t), y^{\Delta}(t)\right)=\frac{\partial L}{\partial y_{k}^{\sigma}}\left(t, y^{\sigma}(t), y^{\Delta}(t)\right), \\
k=1, \ldots, n
\end{array}
$$

for $t \in[a, b]^{\kappa}$.

It is well known that the forward jump operator, $\sigma$, is not delta differentiable for certain time scales. Also, the chain rule as we know it from the classical calculus (i.e., when $\mathbb{T}=\mathbb{R}$ ) is not valid in general. For this reason, we suppose that the time scale $\mathbb{T}$ satisfies the following condition

(H) for each $t \in \mathbb{T}, \sigma(t)=b_{1} t+b_{0}$ for some $b_{1} \in \mathbb{R}^{+}$and $b_{0} \in \mathbb{R}$.

Remark 6. Note that condition $(\mathrm{H})$ implies that $\sigma$ is delta differentiable and $\sigma^{\Delta}(t)=b_{1}, t \in \mathbb{T}^{\kappa}$. Also note that condition (H) describes, in particular, the differential calculus $(\mathbb{T}=\mathbb{R}$, $\left.b_{1}=1, b_{0}=0\right)$, the difference calculus $\left(\mathbb{T}=\mathbb{Z}, b_{1}=1, b_{0}=1\right)$, the $h$-calculus $\left(\mathbb{T}=h \mathbb{Z}:=\{h z: z \in \mathbb{Z}\}, b_{1}=1, b_{0}=h\right.$ for some $h>0)$, and the $q$-calculus $\left(\mathbb{T}=q^{\mathbb{N}_{0}}:=\left\{q^{k}: k \in \mathbb{N}_{0}\right\}\right.$ for some $\left.q>1, b_{1}=q, b_{0}=0\right)$.

Lemma 7 (see [27]). Let $\mathbb{T}$ be a time scale satisfying condition $(H)$. If $f: \mathbb{T} \rightarrow \mathbb{R}$ is two times delta differentiable, then

$$
f^{\sigma \Delta}(t)=b_{1} f^{\Delta \sigma}(t), \quad t \in \mathbb{T}^{\kappa^{2}} .
$$

The next result is also useful for the proofs of our main results.

Lemma 8 (cf. [35]). Assume that the time scale $\mathbb{T}$ satisfies condition $(H), m \in \mathbb{N}$, and $\eta \in C_{\mathrm{rd}}^{2 m}([a, b], \mathbb{R})$ is such that $\eta^{\Delta^{i}}(a)=0$ for all $i=0,1, \ldots, m$. Then, $\eta^{\sigma \Delta^{i-1}}(a)=0$ for each $i=1, \ldots, m$.

\section{Main Results}

In this section, we formulate and prove the second Noether theorem for single and multiple integral variational problems.
3.1. Noether's Second Theorem: Single Delta Integral Case. In this subsection, we suppose that the time scale $\mathbb{T}$ satisfies condition $(\mathrm{H})$ and that $L$ satisfies the assumption of Lemma 4 , for every $y$ and $\eta$. As usual, $\eta^{\Delta^{0}}$ and $\eta^{\sigma^{0}}$ denote $\eta$. Let $m$ be a fixed natural number. We also assume that the time scale interval $[a, b]$ has, at least, $2 m+1$ points.

We begin with some technical results that will be useful in the proofs of Theorems 16 and 22.

Lemma 9 (higher-order fundamental lemma of the calculus of variations). Let $\mathbb{T}$ be a time scale satisfying condition $(H)$ and $f_{0}, f_{1}, \ldots, f_{m} \in C_{\mathrm{rd}}([a, b], \mathbb{R})$. If

$$
\int_{a}^{\rho^{m-1}(b)}\left(\sum_{i=0}^{m} f_{i}(t) \eta^{\sigma^{m-i} \Delta^{i}}(t)\right) \Delta t=0,
$$

for all $\eta \in C_{\mathrm{rd}}^{2 m}([a, b], \mathbb{R})$ such that

$$
\eta(a)=0, \quad \eta\left(\rho^{m-1}(b)\right)=0
$$

$$
\eta^{\Delta^{m-1}}(a)=0, \quad \eta^{\Delta^{m-1}}\left(\rho^{m-1}(b)\right)=0,
$$

then

$$
\sum_{i=0}^{m}(-1)^{i}\left(\frac{1}{b_{1}}\right)^{i(i-1) / 2} f_{i}^{\Delta^{i}}(t)=0, \quad t \in[a, b]^{\kappa^{m}} .
$$

Proof. The proof is similar to the proof of Lemma 16 of [33].

Remark 10. We emphasize that the delta differentiability of the functions $f_{0}, f_{1}, \ldots, f_{m}$ was not assumed in advance.

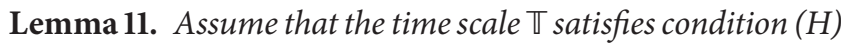
and $\eta \in C_{\mathrm{rd}}^{2 m}([a, b], \mathbb{R})$ is such that,

$$
\eta^{\Delta^{i}}(a)=0, \quad i=0,1, \ldots, m
$$

Then,

$$
\eta^{\sigma^{i}}(a)=0, \quad i=0,1, \ldots, m
$$

Proof. If $a$ is right-dense, the result is trivial. Suppose that $a$ is right-scattered. Since $\eta^{\Delta}(a)=\left(\eta^{\sigma}(a)-\eta(a)\right) /(\sigma(a)-a)=0$ and $\eta(a)=0$, we conclude that $\eta^{\sigma}(a)=0$. Since $\eta^{\Delta^{2}}(a)=$ $\left(\eta^{\Delta}\right)^{\Delta}(a)=\left(\left(\eta^{\Delta}\right)^{\sigma}(a)-\eta^{\Delta}(a)\right) /(\sigma(a)-a)=0$ and $\eta^{\Delta}(a)=0$, then $\left(\eta^{\Delta}\right)^{\sigma}(a)=0$. Using Lemma 7 , we get $\left(\eta^{\sigma}\right)^{\Delta}(a)=0$. Since $\left(\eta^{\sigma}\right)^{\Delta}(a)=\left(\eta^{\sigma^{2}}(a)-\eta^{\sigma}(a)\right) /(\sigma(a)-a)=0$ and $\eta^{\sigma}(a)=0$, we conclude that $\eta^{\sigma^{2}}(a)=0$.

Since $\eta^{\Delta^{3}}(a)=\left(\eta^{\Delta^{2}}\right)^{\Delta}(a)=\left(\left(\eta^{\Delta^{2}}\right)^{\sigma}(a)-\eta^{\Delta^{2}}(a)\right) /(\sigma(a)-$ $a)=0$ and $\eta^{\Delta^{2}}(a)=0$, then $\eta^{\Delta^{2} \sigma}(a)=0$. Using Lemma 7 , we get $\left(\eta^{\sigma}\right)^{\Delta^{2}}(a)=0$. Since $\left(\eta^{\sigma}\right)^{\Delta^{2}}(a)=\left(\eta^{\sigma \Delta \sigma}(a)-\eta^{\sigma \Delta}(a)\right) /(\sigma(a)-$ $a)=0$ and $\eta^{\sigma \Delta}(a)=0$, then $\eta^{\sigma \Delta \sigma}(a)=0$. Lemma 7 proves that $\eta^{\sigma^{2} \Delta}(a)=0$. Since $\eta^{\sigma^{2} \Delta}(a)=\left(\eta^{\sigma^{3}}(a)-\eta^{\sigma^{2}}(a)\right) /(\sigma(a)-a)=0$ and $\eta^{\sigma^{2}}(a)=0$, we get $\eta^{\sigma^{3}}(a)=0$. Repeating recursively this process, we conclude the proof. 
Lemma 12. Assume that the time scale $\mathbb{T}$ satisfies condition $(H)$ and $\eta \in C_{\mathrm{rd}}^{2(m-1)}([a, b], \mathbb{R})$ is such that,

$$
\eta^{\Delta^{i}}(a)=0, \quad i=0,1, \ldots, m-1 .
$$

Then,

$$
\eta^{\sigma \Delta^{m-2}}(a)=\eta^{\sigma^{2} \Delta^{m-3}}(a)=\eta^{\sigma^{3} \Delta^{m-4}}(a)=\ldots=\eta^{\sigma^{m-2} \Delta}(a)=0 .
$$

Proof. If $a$ is right-dense, the result is trivial. Suppose that $a$ is right-scattered. Since $\eta^{\Delta^{m-1}}(a)=\left(\eta^{\Delta^{m-2} \sigma}(a)-\eta^{\Delta^{m-2}}(a)\right) /$ $(\sigma(a)-a)=0$ and $\eta^{\Delta^{m-2}}(a)=0$, then $\eta^{\Delta^{m-2} \sigma}(a)=0$. Using Lemma 7, we get $\eta^{\sigma \Delta^{m-2}}(a)=0$ (or use Lemma 8). Note that $\eta^{\sigma \Delta^{m-2}}(a)=\left(\eta^{\sigma \Delta^{m-3} \sigma}(a)-\eta^{\sigma \Delta^{m-3}}(a)\right) /(\sigma(a)-a)=0$. Lemma 8 shows that $\eta^{\sigma \Delta^{m-3}}(a)=0$; hence, $\eta^{\sigma \Delta^{m-3} \sigma}(a)=0$. Using Lemma 7, we get $\eta^{\sigma^{2} \Delta^{m-3}}(a)=0$. Next, we prove that $\eta^{\sigma^{2} \Delta^{m-4}}$ $(a)=0$. Since $\eta^{\sigma \Delta^{m-3}}(a)=\left(\eta^{\sigma \Delta^{m-4} \sigma}(a)-\eta^{\sigma \Delta^{m-4}}(a)\right) /(\sigma(a)-$ a) $=0$ and $\eta^{\sigma \Delta^{m-4}}(a)=0$ (by Lemma 8), then $\eta^{\sigma \Delta^{m-4} \sigma}(a)=$ 0 . Lemma 7 shows that $\eta^{\sigma^{2} \Delta^{m-4}}(a)=0$. Since $\eta^{\sigma^{2} \Delta^{m-3}}(a)=$ $\left(\eta^{\sigma^{2} \Delta^{m-4} \sigma}(a)-\eta^{\sigma^{2} \Delta^{m-4}}(a)\right) /(\sigma(a)-a)=0$ and $\eta^{\sigma^{2} \Delta^{m-4}}(a)=0$, then $\eta^{\sigma^{2} \Delta^{m-4} \sigma}(a)=0$. Lemma 7 shows that $\eta^{\sigma^{3} \Delta^{m-4}}(a)=0$. Repeating recursively this process, we prove the intended result.

Let $y:=\left(y_{1}, y_{2}, \ldots, y_{n}\right)$. Firstly, we will prove the second Noether theorem without transformation of time. For that consider the following transformations that depend on arbitrary functions $p_{1}, p_{2}, \ldots, p_{r}$ and their delta-derivatives up to order $m$ :

$$
\begin{gathered}
\bar{t}=t \\
\bar{y}_{k}(t)=y_{k}(t)+\sum_{j=1}^{r} T^{k j}\left(p_{j}\right)(t), \quad k=1,2, \ldots, n,
\end{gathered}
$$

where, for each $j=1,2, \ldots, r, p_{j} \in C_{\mathrm{rd}}^{2 m}\left(\left[a, \sigma^{m}(b)\right], \mathbb{R}\right)$,

$$
T^{k j}\left(p_{j}\right):=\sum_{i=0}^{m} g_{i j}^{k} p_{j}^{\sigma^{m-(i+1)} \Delta^{i}}
$$

and $g_{i j}^{k} \in C_{\mathrm{rd}}^{1}([a, b], \mathbb{R})$.

Definition 13. Functional $\mathscr{L}$ is invariant under transformations (16) if, and only if, for all $y \in C_{\mathrm{rd}}^{1}([a, b], \mathbb{R})$, we have

$$
\int_{a}^{b} L\left(t, y^{\sigma}(t), y^{\Delta}(t)\right) \Delta t=\int_{a}^{b} L\left(t, \bar{y}^{\sigma}(t), \bar{y}^{\Delta}(t)\right) \Delta t
$$

Remark 14. Note that the most common definition of invariance (with equality of the integrals for any subinterval $\left[t_{a}, t_{b}\right] \subseteq[a, b]$ with $\left.a, b \in \mathbb{T}\right)$ implies Definition 13 .
Theorem 15 (necessary condition of invariance). If functional $\mathscr{L}$ is invariant under transformations (16), then

$$
\begin{aligned}
\sum_{k=1}^{n} \int_{a}^{b}\left(\frac{\partial L}{\partial y_{k}^{\sigma}}\left(t, y^{\sigma}(t), y^{\Delta}(t)\right) \cdot\left(\sum_{j=1}^{r} T^{k j}\left(p_{j}\right)\right)^{\sigma}(t)\right. \\
\left.\quad+\frac{\partial L}{\partial y_{k}^{\Delta}}\left(t, y^{\sigma}(t), y^{\Delta}(t)\right) \cdot\left(\sum_{j=1}^{r} T^{k j}\left(p_{j}\right)\right)^{\Delta}(t)\right) \Delta t=0 .
\end{aligned}
$$

Proof. Using the definition of invariance and noting that the family of transformations (16) depends upon arbitrary functions $p_{1}, p_{2}, \ldots, p_{r}$, we conclude that, for any real number $\varepsilon$,

$$
\begin{gathered}
\int_{a}^{b} L\left(t, y^{\sigma}(t), y^{\Delta}(t)\right) \Delta t \\
=\int_{a}^{b} L\left(t, y_{1}^{\sigma}(t)+\varepsilon\left(\sum_{j=1}^{r} T^{1 j}\left(p_{j}\right)\right)^{\sigma}(t), \ldots, y_{n}^{\sigma}(t)\right. \\
+\varepsilon\left(\sum_{j=1}^{r} T^{n j}\left(p_{j}\right)\right)^{\sigma}(t) \\
y_{1}^{\Delta}(t)+\varepsilon\left(\sum_{j=1}^{r} T^{1 j}\left(p_{j}\right)\right)^{\Delta}(t), \ldots, y_{n}^{\Delta}(t) \\
\left.+\varepsilon\left(\sum_{j=1}^{r} T^{n j}\left(p_{j}\right)\right)^{\Delta}(t)\right) \Delta t .
\end{gathered}
$$

Differentiating with respect to $\varepsilon$ (use Lemma 4) and taking $\varepsilon=0$, we get equality (19).

Define

$$
E_{k}(L):=\frac{\partial L}{\partial y_{k}^{\sigma}}-\frac{\Delta}{\Delta t} \frac{\partial L}{\partial y_{k}^{\Delta}}, \quad k=1,2, \ldots, n
$$

We will call $E_{k}(L), k=1,2, \ldots, n$, the Euler-Lagrange expressions associated to the Lagrangian $L$.

Theorem 16 (Noether's second theorem without transforming time). If functional $\mathscr{L}$ is invariant under transformations (16), then there exist the following identities:

$$
\begin{array}{r}
\sum_{k=1}^{n} \sum_{i=0}^{m}(-1)^{i}\left(\frac{1}{b_{1}}\right)^{i(i+1) / 2}\left(\left(g_{i j}^{k}\right)^{\sigma} E_{k}(L)\right)^{\Delta^{i}} \equiv 0, \\
j=1,2, \ldots, r .
\end{array}
$$


Proof. Using the necessary condition of invariance (Theorem 15), we conclude that

$$
\begin{aligned}
\sum_{k=1}^{n} \int_{a}^{b}( & \frac{\partial L}{\partial y_{k}^{\sigma}}\left(t, y^{\sigma}(t), y^{\Delta}(t)\right) \cdot\left(\sum_{j=1}^{r} T^{k j}\left(p_{j}\right)\right)^{\sigma}(t) \\
& \left.+\frac{\partial L}{\partial y_{k}^{\Delta}}\left(t, y^{\sigma}(t), y^{\Delta}(t)\right) \cdot\left(\sum_{j=1}^{r} T^{k j}\left(p_{j}\right)\right)^{\Delta}(t)\right) \Delta t=0 .
\end{aligned}
$$

Fix $j=1,2, \ldots, r$. By the arbitrariness of $p_{1}, p_{2}, \ldots, p_{r}$, we can suppose that $p_{h} \equiv 0$ for $h \neq j$. Therefore,

$$
\begin{aligned}
\sum_{k=1}^{n} \int_{a}^{b}( & \frac{\partial L}{\partial y_{k}^{\sigma}}\left(t, y^{\sigma}(t), y^{\Delta}(t)\right) \cdot\left(T^{k j}\left(p_{j}\right)\right)^{\sigma}(t) \\
& \left.+\frac{\partial L}{\partial y_{k}^{\Delta}}\left(t, y^{\sigma}(t), y^{\Delta}(t)\right) \cdot\left(T^{k j}\left(p_{j}\right)\right)^{\Delta}(t)\right) \Delta t=0
\end{aligned}
$$

Integrating by parts, we obtain

$$
\begin{aligned}
\sum_{k=1}^{n}\left(\int_{a}^{b}\left(\frac{\partial L}{\partial y_{k}^{\sigma}}\left(t, y^{\sigma}(t), y^{\Delta}(t)\right)-\frac{\Delta}{\Delta t} \frac{\partial L}{\partial y_{k}^{\Delta}}\left(t, y^{\sigma}(t), y^{\Delta}(t)\right)\right)\right. \\
\cdot\left(T^{k j}\left(p_{j}\right)\right)^{\sigma}(t) \Delta t \\
\left.+\left[\frac{\partial L}{\partial y_{k}^{\Delta}}\left(t, y^{\sigma}(t), y^{\Delta}(t)\right) \cdot T^{k j}\left(p_{j}\right)(t)\right]_{a}^{b}\right)=0 .
\end{aligned}
$$

By the arbitrariness of $p_{j}$, we can restrict to those functions such that

$$
\begin{array}{cc}
p_{j}(a)=0, & p_{j}(b)=0, \\
\vdots & \\
p_{j}^{\Delta^{m-1}}(a)=0, & p_{j}^{\Delta^{m-1}}(b)=0, \\
p_{j}^{\sigma^{-1} \Delta^{m}}(a)=0, & p_{j}^{\sigma^{-1} \Delta^{m}}(b)=0 .
\end{array}
$$

Using Lemmas 8, 11, and 12, we conclude that $T^{k j}\left(p_{j}\right)(a)=0$ and $T^{k j}\left(p_{j}\right)(b)=0, k=1,2, \ldots, n$. Then,

$$
\begin{gathered}
\sum_{k=1}^{n} \int_{a}^{b}\left(\frac{\partial L}{\partial y_{k}^{\sigma}}\left(t, y^{\sigma}(t), y^{\Delta}(t)\right)-\frac{\Delta}{\Delta t} \frac{\partial L}{\partial y_{k}^{\Delta}}\left(t, y^{\sigma}(t), y^{\Delta}(t)\right)\right) \\
\cdot\left(T^{k j}\left(p_{j}\right)\right)^{\sigma}(t) \Delta t=0 ;
\end{gathered}
$$

that is,

$$
\sum_{k=1}^{n} \int_{a}^{b} E_{k}(L)\left(t, y^{\sigma}(t), y^{\Delta}(t)\right) \cdot\left(T^{k j}\left(p_{j}\right)\right)^{\sigma}(t) \Delta t=0 .
$$

Hence,

$$
\begin{aligned}
& \sum_{k=1}^{n} \int_{a}^{b} E_{k}(L)\left(t, y^{\sigma}(t), y^{\Delta}(t)\right) \cdot\left(\sum_{i=0}^{m} g_{i j}^{k} p_{j}^{\sigma^{m-(i+1)} \Delta^{i}}\right)^{\sigma}(t) \Delta t \\
& \quad=0 .
\end{aligned}
$$

Therefore, by Lemma 7, we get

$$
\begin{aligned}
\int_{a}^{b} \sum_{i=0}^{m} \sum_{k=1}^{n} E_{k}(L)\left(t, y^{\sigma}(t), y^{\Delta}(t)\right) \\
\cdot\left(g_{i j}^{k}\right)^{\sigma}(t)\left(\frac{1}{b_{1}}\right)^{i} p_{j}^{\sigma^{m-i} \Delta^{i}}(t) \Delta t=0 .
\end{aligned}
$$

Applying the higher-order fundamental lemma of the calculus of variations (Lemma 9), we obtain

$$
\sum_{i=0}^{m} \sum_{k=1}^{n}(-1)^{i}\left(\frac{1}{b_{1}}\right)^{i(i-1) / 2}\left(E_{k}(L)\left(g_{i j}^{k}\right)^{\sigma}\left(\frac{1}{b_{1}}\right)^{i}\right)^{\Delta^{i}} \equiv 0,
$$

which is equivalent to

$$
\sum_{k=1}^{n} \sum_{i=0}^{m}(-1)^{i}\left(\frac{1}{b_{1}}\right)^{i(i+1) / 2}\left(\left(g_{i j}^{k}\right)^{\sigma} E_{k}(L)\right)^{\Delta^{i}} \equiv 0
$$

proving the desired result.

We present some particular results that follow from Theorem 16 in the case where $\mathbb{T}=\mathbb{R}, \mathbb{T}=h \mathbb{Z}$ (for some $h>0$ ), and $\mathbb{T}=q^{\mathbb{N}_{0}}$ (for some $q>1$ ). If $\mathbb{T}=\mathbb{R}$, then $\sigma(t)=t, f^{\Delta}(t)=\dot{f}(t)$, and we obtain the classical second Noether theorem without transformation of time.

Corollary 17 (cf. [1]). Let $L: \mathbb{R} \times \mathbb{R}^{n} \times \mathbb{R}^{n} \rightarrow \mathbb{R}$ be a $C^{2}$ function. If functional $\mathscr{L}$ defined by

$$
\mathscr{L}[y]=\int_{a}^{b} L(t, y(t), \dot{y}(t)) d t
$$

is invariant under transformations (16) (where $\sigma$ denotes in this context the identity function and $\Delta$ denotes the usual derivative), then there exist the following identities:

$$
\sum_{k=1}^{n} \sum_{i=0}^{m}(-1)^{i}\left[g_{i j}^{k} E_{k}(L)\right]^{(i)} \equiv 0, \quad j=1,2, \ldots, r .
$$

Choosing $h \mathbb{Z}$, we obtain Noether's second theorem without transformation of time for the $h$-calculus.

Corollary 18. Let $h>0, L: h \mathbb{Z} \times \mathbb{R}^{n} \times \mathbb{R}^{n} \rightarrow \mathbb{R}$ and $a, b \in$ $h \mathbb{Z}, a<b$. If functional $\mathscr{L}$ defined by

$$
\mathscr{L}[y]=\sum_{t=a}^{b-h} L\left(t, y(t+h), \Delta_{h}[y](t)\right)
$$

is invariant under transformations (16) (where $\sigma$ denotes in this context the function $\sigma(t)=t+h$ and $\Delta$ denotes the $h$-difference, 
that is, $\left.y^{\Delta}(t)=\Delta_{h}[y](t)=(y(t+h)-y(t)) / h\right)$, then there exist the following identities:

$$
\begin{aligned}
& \sum_{k=1}^{n} \sum_{i=0}^{m}(-1)^{i}\left[g_{i j}^{k}(t+h)\right. \\
&\left.\cdot E_{k}(L)\left(t, y(t+h), \Delta_{h}[y](t)\right)\right]^{\Delta_{h}^{i}}=0 \\
& t \in[a, b-m h], j=1,2, \ldots, r
\end{aligned}
$$

For $h=1$, we obtain the analogue of Noether's second theorem for the difference calculus of variations recently proved in [39]. In the case $\mathbb{T}=q^{\mathbb{N}_{0}}$, we obtain the new result.

Corollary 19. Let $q>1, L: q^{\mathbb{N}_{0}} \times \mathbb{R}^{n} \times \mathbb{R}^{n} \rightarrow \mathbb{R}$ and $a, b \in$ $q^{\mathbb{N}_{0}}, a<b$. If functional $\mathscr{L}$ defined by

$$
\mathscr{L}[y]=\sum_{t=a}^{b / q} L\left(t, y(q t), \Delta_{q}[y](t)\right)
$$

is invariant under transformations (16) (where $\sigma$ denotes in this context the function $\sigma(t)=q t$ and $\Delta$ denotes the $q$-derivative, that is, $\left.y^{\Delta}(t)=\Delta_{q}[y](t)=(y(q t)-y(t)) /(q-1) t\right)$, then there exist the following identities:

$$
\begin{aligned}
& \sum_{k=1}^{n} \sum_{i=0}^{m}(-1)^{i}\left(\frac{1}{q}\right)^{i(i+1) / 2}\left[g_{i j}^{k}(q t)\right. \\
&\left.\cdot E_{k}(L)\left(t, y(q t), \Delta_{q}[y](t)\right)\right]^{\Delta_{q}^{i}}=0, \\
& t \in\left[a, \frac{b}{q^{m}}\right], j=1,2, \ldots, r .
\end{aligned}
$$

In order to prove the second Noether theorem with transformation of time, we will consider that the Lagrangian $L$ is defined for all $t \in \mathbb{R}$ (not only for $t$ from the initial time scale $\mathbb{T}$ ), $L: \mathbb{R} \times \mathbb{R}^{n} \times \mathbb{R}^{n} \rightarrow \mathbb{R}$. Consider the following transformations that depend on arbitrary functions $p_{1}, p_{2}, \ldots, p_{r}$ and their delta-derivatives up to order $m$ :

$$
\begin{gathered}
\bar{t}=t+\sum_{j=1}^{r} H^{j}\left(p_{j}\right)(t), \\
\bar{y}_{k}(\bar{t})=y_{k}(t)+\sum_{j=1}^{r} T^{k j}\left(p_{j}\right)(t), \quad k=1,2, \ldots, n,
\end{gathered}
$$

where, for each $j=1,2, \ldots, r, p_{j} \in C_{\mathrm{rd}}^{2 m}\left(\left[a, \sigma^{m}(b)\right], \mathbb{R}\right)$,

$$
\begin{aligned}
& H^{j}\left(p_{j}\right):=\sum_{i=0}^{m} f_{i j} p_{j}^{\sigma^{m-(i+1)} \Delta^{i}}, \\
& T^{k j}\left(p_{j}\right):=\sum_{i=0}^{m} g_{i j}^{k} p_{j}^{\sigma^{m-(i+1)} \Delta^{i}},
\end{aligned}
$$

$f_{i j} \in C_{\mathrm{rd}}^{1}([a, b], \mathbb{R})$ and $g_{i j}^{k} \in C_{\mathrm{rd}}^{1}([a, b], \mathbb{R})$.
Moreover, we assume that the map

$$
t \longmapsto \alpha(t):=t+\sum_{j=1}^{r} H^{j}\left(p_{j}\right)(t)
$$

is a strictly increasing $C_{\mathrm{rd}}^{1}$ function and its image is again a time scale, $\overline{\mathbb{T}}$. We denote the forward shift operator relative to $\overline{\mathbb{T}}$ by $\bar{\sigma}$ and the delta derivative by $\bar{\Delta}$. We remark that the following holds [40]:

$$
\bar{\sigma} \circ \alpha=\alpha \circ \sigma .
$$

Definition 20. Functional $\mathscr{L}$ is invariant under transformations (39) if, and only if, for all $y \in C_{\mathrm{rd}}^{1}([a, b], \mathbb{R})$, we have

$$
\int_{a}^{b} L\left(t, y^{\sigma}(t), y^{\Delta}(t)\right) \Delta t=\int_{\bar{a}}^{\bar{b}} L\left(\bar{t}, \bar{y}^{\bar{\sigma}}(\bar{t}), \bar{y}^{\bar{\Delta}}(\bar{t})\right) \bar{\Delta} \bar{t} .
$$

We recall the following results that will be very useful in the proof of Theorem 22 .

Theorem 21 (see [37]). Assume that $v: \mathbb{T} \rightarrow \mathbb{R}$ is strictly increasing and $\widetilde{\mathbb{T}}:=v(\mathbb{T})$ is a time scale.

(1) Chain rule: let $\omega: \widetilde{\mathbb{T}} \rightarrow \mathbb{R}$. If $v^{\Delta}(t)$ and $\omega^{\widetilde{\Delta}}(\nu(t))$ exist for all $t \in \mathbb{T}^{\kappa}$, then

$$
(\omega \circ \nu)^{\Delta}=\left(\omega^{\tilde{\Delta}} \circ \nu\right) \nu^{\Delta}
$$

(2) Substitution in the integral: if $f: \widetilde{\mathbb{T}} \rightarrow \mathbb{R}$ is a $C_{\mathrm{rd}}$ function and $v$ is a $C_{\mathrm{rd}}^{1}$ function, then for $a, b \in \mathbb{T}$,

$$
\int_{a}^{b} f(\nu(t)) \nu^{\Delta}(t) \Delta t=\int_{\nu(a)}^{\nu(b)} f(s) \widetilde{\Delta} s .
$$

Now, we are ready to state and prove Noether's second theorem with transformation of time.

Theorem 22 (Noether's second theorem with transformation of time). If functional $\mathscr{L}$ is invariant under transformations (39), then there exist the following identities:

$$
\begin{aligned}
& \sum_{k=1}^{n} \sum_{i=0}^{m}(-1)^{i}\left(\frac{1}{b_{1}}\right)^{i(i+1) / 2}\left[\left(\left(g_{i j}^{k}\right)^{\sigma} E_{k}(L)\right)^{\Delta^{i}}\right. \\
&\left.+\left(\left(f_{i j}\right)^{\sigma}\left(\frac{\partial L}{\partial t}-\frac{\Delta}{\Delta t}\left(L-y_{k}^{\Delta} \frac{\partial L}{\partial y_{k}^{\Delta}}-\mu \frac{\partial L}{\partial t}\right)\right)\right)^{\Delta^{i}}\right] \equiv 0
\end{aligned}
$$

for $j=1,2, \ldots, r$.

Proof. The idea of the proof is to reduce the statement of this result to the one of Theorem 16 using a technique of reparametrization of time: artificially, we will consider $t$ as a dependent variable of the same footing with $y$. 
Let $r \neq 0$ and define

$$
\widetilde{L}(t, s, y, r, v):=L\left(s-\mu(t) r, y, \frac{v}{r}\right) r .
$$

Note that, for $s(t)=t$ and any $y \in C_{\mathrm{rd}}^{1}\left([a, b], \mathbb{R}^{n}\right)$, we have

$$
L\left(t, y^{\sigma}(t), y^{\Delta}(t)\right)=\widetilde{L}\left(t, s^{\sigma}(t), y^{\sigma}(t), s^{\Delta}(t), y^{\Delta}(t)\right) .
$$

Therefore, for $s(t)=t$,

$$
\begin{aligned}
\mathscr{L}[y] & :=\int_{a}^{b} L\left(t, y^{\sigma}(t), y^{\Delta}(t)\right) \Delta t \\
& =\int_{a}^{b} \widetilde{L}\left(t, s^{\sigma}(t), y^{\sigma}(t), s^{\Delta}(t), y^{\Delta}(t)\right) \Delta t:=\widetilde{\mathscr{L}}[s, y] .
\end{aligned}
$$

Note that, for $s(t)=t$,

$$
\begin{aligned}
& \widetilde{\mathscr{L}}[s(\cdot), y(\cdot)] \\
&=\mathscr{L}[y(\cdot)] \\
&=\int_{a}^{b} L\left(t, y^{\sigma}(t), y^{\Delta}(t)\right) \Delta t \\
&=\int_{\alpha(a)}^{\alpha(b)} L\left(\bar{t},(\bar{y} \circ \bar{\sigma})(\bar{t}), \bar{y}^{\bar{\Delta}}(\bar{t})\right) \bar{\Delta} \bar{t} \\
&= \int_{a}^{b} L\left(\alpha(t),(\bar{y} \circ \bar{\sigma} \circ \alpha)(t), \bar{y}^{\bar{\Delta}}(\alpha(t))\right) \alpha^{\Delta}(t) \Delta t \\
&= \int_{a}^{b} L\left(\alpha(t),(\bar{y} \circ \alpha \circ \sigma)(t), \frac{(\bar{y} \circ \alpha)^{\Delta}(t)}{\alpha^{\Delta}(t)}\right) \alpha^{\Delta}(t) \Delta t \\
&= \int_{a}^{b} L\left(\alpha^{\sigma}(t)-\mu(t) \alpha^{\Delta}(t),(\bar{y} \circ \alpha)^{\sigma}(t), \frac{(\bar{y} \circ \alpha)^{\Delta}(t)}{\alpha^{\Delta}(t)}\right) \\
& \cdot \alpha^{\Delta}(t) \Delta t \\
&= \int_{a}^{b} \widetilde{L}\left(t, \alpha^{\sigma}(t),(\bar{y} \circ \alpha)^{\sigma}(t), \alpha^{\Delta}(t),(\bar{y} \circ \alpha)^{\Delta}(t)\right) \Delta t \\
&= \widetilde{\mathscr{L}}[\alpha(\cdot),(\bar{y} \circ \alpha)(\cdot)] .
\end{aligned}
$$

Let $H(t, y(t)):=\alpha(t)$ and $T=\left(T^{1}, T^{2}, \ldots, T^{n}\right)$ where

$$
T^{k}(t, y(t)):=y_{k}(t)+\sum_{j=1}^{r} T^{k j}\left(p_{j}\right)(t), \quad k=1,2, \ldots, n .
$$

Then, for $s(t)=t$,

$$
\begin{aligned}
(\alpha(t),(\bar{y} \circ \alpha)(t)) & =(\bar{t}, \bar{y}(\bar{t})) \\
& =(H(t, y(t)), T(t, y(t))) \\
& =(H(s(t), y(t)), T(s(t), y(t))) .
\end{aligned}
$$

Hence, using (50) and (52), we get

$$
\widetilde{\mathscr{L}}[s(\cdot), y(\cdot)]=\widetilde{\mathscr{L}}[H(s(\cdot), y(\cdot)), T(s(\cdot), y(\cdot))] .
$$

This means that $\widetilde{\mathscr{L}}$ is invariant on

$$
\widetilde{U}=\left\{(s, y) \mid s(t)=t \wedge y \in C_{\text {rd }}^{1}\left([a, b], \mathbb{R}^{n}\right)\right\}
$$

under the group of state transformations

$$
(\bar{s}, \bar{y})=(H(s, y), T(s, y))
$$

in the sense of Definition 13.

Using Theorem 16, we can conclude that there exist the following $r$ identities $(j=1,2, \ldots, r)$

$$
\begin{aligned}
\sum_{k=1}^{n} \sum_{i=0}^{m}( & -1)^{i}\left(\frac{1}{b_{1}}\right)^{i(i+1) / 2}\left(\left(g_{i j}^{k}\right)^{\sigma} E_{k}(\widetilde{L})\right)^{\Delta^{i}} \\
& +\sum_{i=0}^{m}(-1)^{i}\left(\frac{1}{b_{1}}\right)^{i(i+1) / 2}\left(\left(f_{i j}\right)^{\sigma} E_{s}(\widetilde{L})\right)^{\Delta^{i}} \equiv 0,
\end{aligned}
$$

where we denote $E_{s}(\widetilde{L}):=\left(\partial \widetilde{L} / \partial s^{\sigma}\right)-(\Delta / \Delta t)\left(\partial \widetilde{L} / \partial s^{\Delta}\right)$.

Note that, for $s(t)=t$,

$$
\begin{aligned}
& \frac{\partial \widetilde{L}}{\partial s^{\sigma}}(\left.t, s^{\sigma}(t), y^{\sigma}(t), s^{\Delta}(t), y^{\Delta}(t)\right) \\
&= \frac{\partial L}{\partial t}\left(s^{\sigma}(t)-\mu(t) s^{\Delta}(t), y^{\sigma}(t), \frac{y^{\Delta}(t)}{s^{\Delta}(t)}\right) s^{\Delta}(t), \\
& \frac{\partial \widetilde{L}}{\partial s^{\Delta}}\left(t, s^{\sigma}(t), y^{\sigma}(t), s^{\Delta}(t), y^{\Delta}(t)\right) \\
&=L\left(s^{\sigma}(t)-\mu(t) s^{\Delta}(t), y^{\sigma}(t), \frac{y^{\Delta}(t)}{s^{\Delta}(t)}\right) \\
&-\sum_{k=1}^{n} \frac{y_{k}^{\Delta}(t)}{s^{\Delta}(t)} \frac{\partial L}{\partial y_{k}^{\Delta}}\left(s^{\sigma}(t)-\mu(t) s^{\Delta}(t), y^{\sigma}(t), \frac{y^{\Delta}(t)}{s^{\Delta}(t)}\right) \\
& \quad-\frac{\partial L}{\partial t}\left(s^{\sigma}(t)-\mu(t) s^{\Delta}(t), y^{\sigma}(t), \frac{y^{\Delta}(t)}{s^{\Delta}(t)}\right) \\
& \quad \cdot \mu(t) s^{\Delta}(t) .
\end{aligned}
$$

Hence, for $s(t)=t$,

$$
\begin{aligned}
E_{s}(\widetilde{L}) & \left(t, s^{\sigma}(t), y^{\sigma}(t), s^{\Delta}(t), y^{\Delta}(t)\right) \\
= & \frac{\partial L}{\partial t}\left(t, y^{\sigma}(t), y^{\Delta}(t)\right) \\
& -\frac{\Delta}{\Delta t}\left(L\left(t, y^{\sigma}(t), y^{\Delta}(t)\right)\right.
\end{aligned}
$$

$$
\begin{aligned}
& -\sum_{k=1}^{n} y_{k}^{\Delta}(t) \frac{\partial L}{\partial y_{k}^{\Delta}}\left(t, y^{\sigma}(t), y^{\Delta}(t)\right) \\
& \left.-\mu(t) \frac{\partial L}{\partial t}\left(t, y^{\sigma}(t), y^{\Delta}(t)\right)\right) .
\end{aligned}
$$


Also note that, for $s(t)=t$ and $k=1,2, \ldots, n$,

$$
\begin{gathered}
E_{k}(\widetilde{L})\left(t, s^{\sigma}(t), y^{\sigma}(t), s^{\Delta}(t), y^{\Delta}(t)\right) \\
=E_{k}(L)\left(t, y^{\sigma}(t), y^{\Delta}(t)\right) .
\end{gathered}
$$

Substituting the above equalities into (56), we conclude the desired result:

$$
\begin{aligned}
& \sum_{k=1}^{n} \sum_{i=0}^{m}(-1)^{i}\left(\frac{1}{b_{1}}\right)^{i(i+1) / 2} {\left[\left(\left(g_{i j}^{k}\right)^{\sigma} E_{k}(L)\right)^{\Delta^{i}}\right.} \\
&\left.+\left(\left(f_{i j}\right)^{\sigma}\left(\frac{\partial L}{\partial t}-\frac{\Delta}{\Delta t}\left(L-y_{k}^{\Delta} \frac{\partial L}{\partial y_{k}^{\Delta}}-\mu \frac{\partial L}{\partial t}\right)\right)\right)^{\Delta^{i}}\right] \equiv 0
\end{aligned}
$$

for $j=1,2, \ldots, r$.

Remark 23. Define

$$
E_{k}^{s}(L):=\frac{\partial L}{\partial t}-\frac{\Delta}{\Delta t}\left(L-\sum_{k=1}^{n} y_{k}^{\Delta} \frac{\partial L}{\partial y_{k}^{\Delta}}-\mu \frac{\partial L}{\partial t}\right),
$$

for $k=1,2, \ldots, n$. Then, $E_{k}^{s}(L)=0$ are the second EulerLagrange equations for problem (1) [36]. Therefore, expression (46) provides "dependencies" between two types of the Euler-Lagrange expressions.

Note that if $\mathbb{T}=\mathbb{R}$, Noether's identity (46) simplifies because

$$
\begin{aligned}
\frac{\partial L}{\partial t}- & \frac{\Delta}{\Delta t}\left(L-\sum_{k=1}^{n} y_{k}^{\Delta} \frac{\partial L}{\partial y_{k}^{\Delta}}-\mu \frac{\partial L}{\partial t}\right) \\
& =\frac{\partial L}{\partial t}-\frac{d}{d t}\left(L-\sum_{k=1}^{n} \dot{y}_{k} \frac{\partial L}{\partial \dot{y}_{k}}\right)=-\sum_{k=1}^{n} \dot{y}_{k} E_{k}(L),
\end{aligned}
$$

and we obtain the following corollary.

Corollary 24 (classical Noether's second theorem, cf. [1]). If functional $\mathscr{L}$ defined by

$$
\mathscr{L}[y]=\int_{a}^{b} L(t, y(t), \dot{y}(t)) d t
$$

is invariant under transformations (39) (where $\sigma$ denotes in this context the identity function and $\Delta$ denotes the usual derivative), then there exist the following identities:

$$
\begin{array}{r}
\sum_{k=1}^{n} \sum_{i=0}^{m}(-1)^{i}\left[\left(g_{i j}^{k} E_{k}(L)\right)^{(i)}-\left(f_{i j} \cdot \dot{y}_{k} E_{k}(L)\right)^{(i)}\right] \equiv 0, \\
j=1,2, \ldots, r .
\end{array}
$$

In the case $\mathbb{T}=h \mathbb{Z}$ (for some $h>0$ ), we obtain from Theorem 22 the second Noether theorem for the $h$-calculus; whereas if $\mathbb{T}=q^{\mathbb{N}_{0}}$ (for some $q>1$ ), we get the second Noether theorem for the $q$-calculus.
3.2. Noether's Second Theorem: Multiple Delta Integral Case. In this subsection, we extend the second Noether theorem (without transformation of time) to multiple integral variational problems in the time scale setting. For simplicity of presentation, we prove the result for the case of two independent variables and transformations that depend on an arbitrary function and its first-order partial delta derivatives. Clearly, our result can be generalized for $n$ independent variables and $r$ arbitrary functions and their higher-order partial delta derivatives.

For the convenience of the reader, we recall notions and results that are needed in the sequel. A general introduction to differential calculus and integration theory for multivariable functions on time scales is presented, respectively, in [41] (see also [42]) and [43]. For the double integral calculus of variations on time scales, we refer the reader to [44].

Let $\mathbb{T}_{1}$ and $\mathbb{T}_{2}$ be two given time scales. For $i=1,2$, denote by $\sigma_{i}$ and $\Delta_{i}$ the forward jump operator and the delta derivative on $\mathbb{T}_{i}$, respectively. Let $C_{\mathrm{rd}}^{(1)}$ denote the set of all continuous functions defined on $\mathbb{T}_{1} \times \mathbb{T}_{2}$ for which both the $\Delta_{1}$-partial derivative and the $\Delta_{2}$-partial derivative exist and are of class $C_{\text {rd }}$ (for a definition see [44]).

Let $\Omega \subseteq \mathbb{T}_{1} \times \mathbb{T}_{2}$ be an $\omega$-type set and let $\Gamma$ be its positively fence (see [44]). Denote

$$
\Omega^{\circ}:=\left\{(x, y) \in \Omega:\left(\sigma_{1}(x), \sigma_{2}(y)\right) \in \Omega\right\} .
$$

Let a function $L(x, y, u, p, q)$, where $(x, y) \in \Omega \cup \Gamma$ and $(u, p, q) \in \mathbb{R}^{3 n}$, be given. We will suppose that $L$ is continuous, together with its partial delta derivatives of first and second order with respect to $x, y$ and partial usual derivatives of the first and second order with respect to $u, p, q$. In what follows, $u^{\Delta_{1}}$ and $u^{\Delta_{2}}$ denote, respectively, $\partial u / \Delta_{1} x$ and $\partial u / \Delta_{2} y$.

Consider the following optimization problem:

$$
\begin{array}{r}
\mathscr{L}[u]=\iint_{\Omega} L\left(x, y, u\left(\sigma_{1}(x), \sigma_{2}(y)\right), u^{\Delta_{1}}\left(x, \sigma_{2}(y)\right),\right. \\
\left.u^{\Delta_{2}}\left(\sigma_{1}(x), y\right)\right) \Delta_{1} x \Delta_{2} y \rightarrow \text { extremize },
\end{array}
$$

where the set of admissible functions are

$$
\mathscr{D}=\left\{u \mid u: \Omega \cup \Gamma \longrightarrow \mathbb{R}^{n}, u \in C_{\mathrm{rd}}^{(1)}, u=g \text { on } \Gamma\right\},
$$

where $g$ is a fixed function defined and continuous on the fence $\Gamma$ of $\Omega$.

As noticed in [44], for the variational problem (66) being well posed, we have to assume that there exists at least one admissible function $u_{0} \in \mathscr{D}$ because it is possible to choose a continuous function $g$ such that no function $u$ is admissible. Note that if there exists an admissible function $u_{0}$, then the set $\mathscr{D}$ contains a set of functions of the form $u=u_{0}+\eta$, where $\eta: \Omega \cup \Gamma \rightarrow \mathbb{R}^{n}$ is $C_{\mathrm{rd}}^{(1)}$ and $\eta=0$ on $\Gamma$. Any such $\eta$ is called an admissible variation for problem (66).

Definition 25. We say that $u_{*} \in \mathscr{D}$ is a local minimizer (resp., local maximizer) for problem (66) if there exists $\delta>0$ such that

$$
\mathscr{L}\left[u_{*}\right] \leq \mathscr{L}[u]\left(\text { resp., } \mathscr{L}\left[u_{*}\right] \geq \mathscr{L}[u]\right),
$$


for all $u \in \mathscr{D}$ with

$$
\begin{aligned}
\left\|u-u_{*}\right\|:= & \sup _{(x, y) \in \Omega \cup \Gamma}\left|u(x, y)-u_{*}(x, y)\right| \\
& +\sup _{(x, y) \in \Omega}\left|u^{\Delta_{1}}\left(x, \sigma_{2}(y)\right)-u_{*}^{\Delta_{1}}\left(x, \sigma_{2}(y)\right)\right| \\
& +\sup _{(x, y) \in \Omega}\left|u^{\Delta_{2}}\left(\sigma_{1}(x), y\right)-u_{*}^{\Delta_{2}}\left(\sigma_{1}(x), y\right)\right|<\delta,
\end{aligned}
$$

where $|\cdot|$ denotes a norm in $\mathbb{R}^{n}$.

We recall the following results which will play an important role in the proofs of our results.

Theorem 26 (Green's theorem [45]). If the functions $M$ and $N$ are continuous and have continuous partial delta derivatives $\partial M / \Delta_{2} y$ and $\partial N / \Delta_{1} x$ on $\Omega \cup \Gamma$, then

$$
\iint_{\Omega}\left(\frac{\partial N}{\Delta_{1} x}-\frac{\partial M}{\Delta_{2} y}\right) \Delta_{1} x \Delta_{2} y=\int_{\Gamma} M d^{*} x+N d^{*} y,
$$

where the "star line integrals" on the right side in (70) denote the sum of line delta integrals taken over the line segment constituents of $\Gamma$ directed to the right or upwards and line nabla integrals taken over the line segment constituents of $\Gamma$ directed to the left or downwards.

Lemma 27 (fundamental lemma of the double variational calculus [44]). If $M$ is continuous on $\Omega \cup \Gamma$ with

$$
\iint_{\Omega} M(x, y) \eta\left(\sigma_{1}(x), \sigma_{2}(y)\right) \Delta_{1} x \Delta_{2} y=0
$$

for any admissible variation $\eta$, then

$$
M(x, y)=0 \quad \forall(x, y) \in \Omega^{\circ} .
$$

Theorem 28 (Euler-Lagrange equation of the double variational calculus [44]). Suppose that an admissible function $u_{*}$ provides a local minimum for $\mathscr{L}$ and that $u_{*}$ has continuous partial delta derivatives of the second order. Then $u_{*}$ satisfies the Euler-Lagrange equation

$$
\frac{\partial L}{\partial u}(\cdot)-\frac{\partial}{\Delta_{1} x} \frac{\partial L}{\partial p}(\cdot)-\frac{\partial}{\Delta_{2} y} \frac{\partial L}{\partial q}(\cdot)=0,
$$

where $(\cdot)=\left(x, y, u\left(\sigma_{1}(x), \sigma_{2}(y)\right), u^{\Delta_{1}}\left(x, \sigma_{2}(y)\right), u^{\Delta_{2}}\left(\sigma_{1}(x)\right.\right.$, y)) for $(x, y) \in \Omega^{\circ}$.

Let us denote by $\rho_{1}$ and $\rho_{2}$ the backward jump operator of $\mathbb{T}_{1}$ and $\mathbb{T}_{2}$, respectively. In what follows, we will suppose that $\mathbb{T}_{1}$ and $\mathbb{T}_{2}$ are such that

$$
\begin{array}{ll}
\sigma_{1}\left(\rho_{1}(x)\right)=x, & \forall x \in\left(\mathbb{T}_{1}\right)_{\kappa}, \\
\sigma_{2}\left(\rho_{2}(y)\right)=y, & \forall y \in\left(\mathbb{T}_{2}\right)_{\kappa},
\end{array}
$$

where $\mathbb{T}_{\kappa}:=\mathbb{T} \backslash\{m\}$ if $\mathbb{T}$ has a right-scattered minimum $m$; otherwise, $\mathbb{T}_{\kappa}=\mathbb{T}$. We recall the fact that $\mathbb{R}, h \mathbb{Z}$ (for some $h>0), q^{\mathbb{N}_{0}}$ (for some $q>1$ ), and many other interesting time scales satisfy property $(74)$.

Let $u(x, y)=\left(u_{1}(x, y), u_{2}(x, y), \ldots, u_{n}(x, y)\right)$ and consider the following transformations that depend on an arbitrary continuous function $p$ and the partial delta derivatives of $p$ :

$$
\begin{gathered}
\bar{x}=x \\
\bar{y}=y \\
\bar{u}_{k}(\bar{x}, \bar{y})=u_{k}(x, y)+T^{k}(p)(x, y),
\end{gathered}
$$

where, for each $k=1,2, \ldots, n$,

$$
\begin{aligned}
T^{k}(p)(x, y):= & a_{0}^{k}(x, y) p(x, y) \\
& +a_{1}^{k}(x, y) \frac{\partial}{\Delta_{1} x} p\left(\rho_{1}(x), y\right) \\
& +a_{2}^{k}(x, y) \frac{\partial}{\Delta_{2} y} p\left(x, \rho_{2}(y)\right),
\end{aligned}
$$

$a_{0}, a_{1}$, and $a_{2}$ are $C^{1}$ functions and we assume that $p$ has continuous partial delta derivatives of the first and second order.

Definition 29. Functional $\mathscr{L}$ is invariant under transformations (75) if, and only if, for all $u \in \mathscr{D}$, we have

$$
\begin{gathered}
\iint_{\Omega} L\left(x, y, u\left(\sigma_{1}(x), \sigma_{2}(y)\right), u^{\Delta_{1}}\left(x, \sigma_{2}(y)\right),\right. \\
\left.u^{\Delta_{2}}\left(\sigma_{1}(x), y\right)\right) \Delta_{1} x \Delta_{2} y \\
=\iint_{\Omega} L\left(x, y, \bar{u}\left(\sigma_{1}(x), \sigma_{2}(y)\right), \bar{u}^{\Delta_{1}}\left(x, \sigma_{2}(y)\right),\right. \\
\left.\bar{u}^{\Delta_{2}}\left(\sigma_{1}(x), y\right)\right) \Delta_{1} x \Delta_{2} y .
\end{gathered}
$$

In what follows, we use the notations

$$
\begin{gathered}
T^{k}\left(p^{\sigma}\right)(x, y):=T^{k}\left(\sigma_{1}(x), \sigma_{2}(y)\right), \\
T^{k}\left(p^{\sigma_{1}}\right)(x, y):=T^{k}\left(\sigma_{1}(x), y\right), \\
T^{k}\left(p^{\sigma_{2}}\right)(x, y):=T^{k}\left(x, \sigma_{2}(y)\right) .
\end{gathered}
$$

Using similar arguments as the ones used in the proof of Theorem 15, we can prove the following result.

Theorem 30 (necessary condition of invariance). If functional $\mathscr{L}$ is invariant under transformations (75), then

$$
\begin{gathered}
\sum_{k=1}^{n} \iint_{\Omega}\left(\frac{\partial L}{\partial u_{k}^{\sigma}} \cdot T^{k}\left(p^{\sigma}\right)+\frac{\partial L}{\partial u_{k}^{\Delta_{1}}} \frac{\partial}{\Delta_{1} x} T^{k}\left(p^{\sigma_{2}}\right)\right. \\
\left.+\frac{\partial L}{\partial u_{k}^{\Delta_{2}}} \frac{\partial}{\Delta_{2} y} T^{k}\left(p^{\sigma_{1}}\right)\right) \Delta_{1} x \Delta_{2} y=0 .
\end{gathered}
$$


Define

$$
\widehat{E}_{k}(L):=\frac{\partial L}{\partial u_{k}^{\sigma}}-\frac{\partial}{\Delta_{1} x} \frac{\partial L}{\partial u_{k}^{\Delta_{1}}}-\frac{\partial}{\Delta_{2} y} \frac{\partial L}{\partial u_{k}^{\Delta_{2}}}, \quad k=1,2, \ldots, n .
$$

We call $\widehat{E}_{k}(L), k=1,2, \ldots, n$, the Euler-Lagrange expressions associated to the Lagrangian $L$ relative to problem (66).

The following lemmas will be used in the proof of Theorem 34.

Lemma 31. If $\mathscr{L}$ is invariant under transformations (75), then

$$
\sum_{k=1}^{n} \iint_{\Omega} \widehat{E}_{k}(L) \cdot T^{k}\left(p^{\sigma}\right) \Delta_{1} x \Delta_{2} y=0
$$

Proof. Fix $i \in\{1,2, \ldots, n\}$. Observe that

$$
\begin{gathered}
\iint_{\Omega}\left(\frac{\partial L}{\partial u_{i}^{\Delta_{1}}} \frac{\partial}{\Delta_{1} x} T^{i}\left(p^{\sigma_{2}}\right)+\frac{\partial L}{\partial u_{i}^{\Delta_{2}}} \frac{\partial}{\Delta_{2} y} T^{i}\left(p^{\sigma_{1}}\right)\right) \Delta_{1} x \Delta_{2} y \\
=\iint_{\Omega}\left[\frac{\partial}{\Delta_{1} x}\left(\frac{\partial L}{\partial u_{i}^{\Delta_{1}}} \cdot T^{i}\left(p^{\sigma_{2}}\right)\right)\right. \\
\left.+\frac{\partial}{\Delta_{2} y}\left(\frac{\partial L}{\partial u_{i}^{\Delta_{2}}} \cdot T^{i}\left(p^{\sigma_{1}}\right)\right)\right] \Delta_{1} x \Delta_{2} y \\
-\iint_{\Omega}\left[\frac{\partial}{\Delta_{1} x} \frac{\partial L}{\partial u_{i}^{\Delta_{1}}} \cdot T^{i}\left(p^{\sigma}\right)+\frac{\partial}{\Delta_{2} y} \frac{\partial L}{\partial u_{i}^{\Delta_{2}}} \cdot T^{i}\left(p^{\sigma}\right)\right] \Delta_{1} x \Delta_{2} y .
\end{gathered}
$$

Using Green's theorem we get

$$
\begin{aligned}
\iint_{\Omega} & {\left[\frac{\partial}{\Delta_{1} x}\left(\frac{\partial L}{\partial u_{i}^{\Delta_{1}}} \cdot T^{i}\left(p^{\sigma_{2}}\right)\right)\right.} \\
& \left.+\frac{\partial}{\Delta_{2} y}\left(\frac{\partial L}{\partial u_{i}^{\Delta_{2}}} \cdot T^{i}\left(p^{\sigma_{1}}\right)\right)\right] \Delta_{1} x \Delta_{2} y \\
= & \int_{\Gamma} \frac{\partial L}{\partial u_{i}^{\Delta_{1}}} \cdot T^{i}\left(p^{\sigma_{2}}\right) d^{*} y-\frac{\partial L}{\partial u_{i}^{\Delta_{2}}} \cdot T^{i}\left(p^{\sigma_{1}}\right) d^{*} x .
\end{aligned}
$$

$$
\begin{gathered}
\left.p\left(x, \sigma_{2}(y)\right)\right|_{\Gamma}=0, \\
\left.p\left(\sigma_{1}(x), y\right)\right|_{\Gamma}=0, \\
\left.\frac{\partial}{\Delta_{1} x} p\left(\rho_{1}(x), \sigma_{2}(y)\right)\right|_{\Gamma}=0, \\
\left.\frac{\partial}{\Delta_{1} x} p(x, y)\right|_{\Gamma}=0, \\
\left.\frac{\partial}{\Delta_{2} y} p(x, y)\right|_{\Gamma}=0, \\
\left.\frac{\partial}{\Delta_{2} y} p\left(\sigma_{1}(x), \rho_{2}(y)\right)\right|_{\Gamma}=0,
\end{gathered}
$$

and therefore,

$$
\begin{aligned}
\iint_{\Omega}\left[\frac{\partial}{\Delta_{1} x}\left(\frac{\partial L}{\partial u_{i}^{\Delta_{1}}} \cdot T^{i}\left(p^{\sigma_{2}}\right)\right)\right. \\
\left.\quad+\frac{\partial}{\Delta_{2} y}\left(\frac{\partial L}{\partial u_{i}^{\Delta_{2}}} \cdot T^{i}\left(p^{\sigma_{1}}\right)\right)\right] \Delta_{1} x \Delta_{2} y=0 .
\end{aligned}
$$

By Theorem 30, we obtain

$$
\begin{array}{r}
\sum_{k=1}^{n} \iint_{\Omega}\left(\frac{\partial L}{\partial u_{k}^{\sigma}} \cdot T^{k}\left(p^{\sigma}\right)-\frac{\partial}{\Delta_{1} x} \frac{\partial L}{\partial u_{k}^{\Delta_{1}}} \cdot T^{k}\left(p^{\sigma}\right)\right. \\
\left.-\frac{\partial}{\Delta_{2} y} \frac{\partial L}{\partial u_{k}^{\Delta_{2}}} \cdot T^{k}\left(p^{\sigma}\right)\right) \Delta_{1} x \Delta_{2} y=0
\end{array}
$$

which proves that $\sum_{k=1}^{n} \iint_{\Omega} \widehat{E}_{k}(L) \cdot T^{k}\left(p^{\sigma}\right) \Delta_{1} x \Delta_{2} y=0$.

Lemma 32. For each $k=1,2, \ldots, n$,

$$
\begin{aligned}
\iint_{\Omega} q \cdot T^{k}\left(p^{\sigma}\right) \Delta_{1} x \Delta_{2} y \\
\quad=\iint_{\Omega}\left(q a_{0}^{k}-\frac{\partial}{\Delta_{1} x}\left(q a_{1}^{k}\right)-\frac{\partial}{\Delta_{2} y}\left(q a_{2}^{k}\right)\right) \cdot p^{\sigma} \Delta_{1} x \Delta_{2} y
\end{aligned}
$$

holds.

Proof. Note that

$$
\begin{aligned}
& \iint_{\Omega} q \cdot T^{k}\left(p^{\sigma}\right) \Delta_{1} x \Delta_{2} y \\
& =\iint_{\Omega}\left[q a_{0}^{k}(x, y) p^{\sigma}(x, y)\right. \\
& +q a_{1}^{k}(x, y) \frac{\partial}{\Delta_{1} x} p\left(x, \sigma_{2}(y)\right) \\
& \left.+q a_{2}^{k}(x, y) \frac{\partial}{\Delta_{2} y} p\left(\sigma_{1}(x), y\right)\right] \Delta_{1} x \Delta_{2} y
\end{aligned}
$$$$
\iint_{\Omega}\left[q a_{1}^{k}(x, y) \frac{\partial}{\Delta_{1} x} p\left(x, \sigma_{2}(y)\right)\right.
$$$$
\left.+q a_{2}^{k}(x, y) \frac{\partial}{\Delta_{2} y} p\left(\sigma_{1}(x), y\right)\right] \Delta_{1} x \Delta_{2} y
$$$$
=\iint_{\Omega}\left[\frac{\partial}{\Delta_{1} x}\left(q a_{1}^{k}(x, y) p\left(x, \sigma_{2}(y)\right)\right)\right.
$$$$
\left.+\frac{\partial}{\Delta_{2} y}\left(q a_{2}^{k}(x, y) p\left(\sigma_{1}(x), y\right)\right)\right] \Delta_{1} x \Delta_{2} y
$$$$
-\iint_{\Omega}\left[\frac{\partial}{\Delta_{1} x}\left(q a_{1}^{k}(x, y)\right) \cdot p\left(\sigma_{1}(x), \sigma_{2}(y)\right)\right.
$$$$
\left.+\frac{\partial}{\Delta_{2} y}\left(q a_{2}^{k}(x, y)\right) \cdot p\left(\sigma_{1}(x), \sigma_{2}(y)\right)\right] \Delta_{1} x \Delta_{2} y .
$$ 
Using Green's theorem, we can conclude that

$$
\begin{aligned}
\iint_{\Omega} & {\left[\frac{\partial}{\Delta_{1} x}\left(q a_{1}^{k}(x, y) p\left(x, \sigma_{2}(y)\right)\right)\right.} \\
& \left.+\frac{\partial}{\Delta_{2} y}\left(q a_{2}^{k}(x, y) p\left(\sigma_{1}(x), y\right)\right)\right] \Delta_{1} x \Delta_{2} y=0 .
\end{aligned}
$$

Hence,

$$
\begin{aligned}
\iint_{\Omega} q \cdot T^{k}\left(p^{\sigma}\right) \Delta_{1} x \Delta_{2} y \\
=\iint_{\Omega}\left[q a_{0}^{k} \cdot p^{\sigma}-\frac{\partial}{\Delta_{1} x}\left(q a_{1}^{k}\right) \cdot p^{\sigma}\right. \\
\left.\quad-\frac{\partial}{\Delta_{2} y}\left(q a_{2}^{k}\right) \cdot p^{\sigma}\right] \Delta_{1} x \Delta_{2} y
\end{aligned}
$$

proving the desired result.

Remark 33. Lemma 32 shows that we can define an adjoint operator of $T^{k}, \widetilde{T}^{k}$, by

$$
\widetilde{T}^{k}(q)=q a_{0}^{k}-\frac{\partial}{\Delta_{1} x}\left(q a_{1}^{k}\right)-\frac{\partial}{\Delta_{2} y}\left(q a_{2}^{k}\right) .
$$

We are now ready to state and prove the Noether second theorem without transformation of time for multiple integral problems on time scales.

Theorem 34 (Noether's second theorem without transforming time). If functional $\mathscr{L}$ is invariant under transformations (75), then

$$
\sum_{k=1}^{n} \widetilde{T}^{k}\left(\widehat{E}_{k}(L)\right) \equiv 0 \quad \text { on } \Omega^{\circ}
$$

where $\widehat{E}_{k}(L)$ are the $n$ Euler-Lagrange expressions and $\widetilde{T}^{k}$ is the adjoint operator of $T^{k}$.

Proof. Using Lemmas 31 and 32, we conclude that if $\mathscr{L}$ is invariant under transformations (75), then

$$
\begin{aligned}
& \sum_{k=1}^{n} \iint_{\Omega} \widehat{E}_{k}(L) \cdot T^{k}\left(p^{\sigma}\right) \Delta_{1} x \Delta_{2} y \\
& \quad=\sum_{k=1}^{n} \iint_{\Omega} \widetilde{T}^{k}\left(\widehat{E}_{k}(L)\right) \cdot p^{\sigma} \Delta_{1} x \Delta_{2} y=0
\end{aligned}
$$

where $\widetilde{T}^{k}$ is the adjoint operator of $T^{k}$. Applying the fundamental lemma of the double variational calculus (Lemma 27), we get

$$
\sum_{k=1}^{n} \widetilde{T}^{k}\left(\widehat{E}_{k}(L)\right) \equiv 0 \quad \text { on } \Omega^{\circ}
$$

proving the desired result.
Corollary 35 (classical Noether's second theorem for double integrals problems, cf. [1]). Let $\Omega \subseteq \mathbb{R}^{2}$ be an $\omega$-type set and let $\Gamma$ be its positive fence. Let $L(x, y, u, p, q)$ be a function of class $C^{2},(x, y) \in \Omega \cup \Gamma, u=\left(u_{1}, u_{2}, \ldots, u_{n}\right)$. If functional $\mathscr{L}$ defined by

$$
\mathscr{L}[y]=\iint_{\Omega} L\left(x, y, u(x, y), \frac{\partial u}{\partial x}(x, y), \frac{\partial u}{\partial y}(x, y)\right) d x d y
$$

is invariant under transformations (75) (where $\rho_{1}$ and $\rho_{2}$ denote in this context the identity function, and $\Delta_{1}$ and $\Delta_{2}$ denote the usual derivative), then

$$
\sum_{k=1}^{n} \widetilde{T}^{k}\left(\widehat{E}_{k}(L)\right) \equiv 0 \quad \text { on } \Omega
$$

where

$$
\widehat{E}_{k}(L):=\frac{\partial L}{\partial u_{k}}-\frac{\partial}{\partial x} \frac{\partial L}{\partial p_{k}}-\frac{\partial}{\partial y} \frac{\partial L}{\partial q_{k}}, \quad k=1,2, \ldots, n
$$

and $\widetilde{T}^{k}$ is the adjoint operator of $T^{k}$.

Similarly to the single delta integral case choosing $\mathbb{T}=$ $h \mathbb{Z}$ (for some $h>0$ ), we obtain from Theorem 34 the second Noether theorem for the double variational $h$-calculus; whereas choosing $\mathbb{T}=q^{\mathbb{N}_{0}}$ (for some $q>1$ ), we obtain the second Noether theorem for the double variational $q$ calculus.

\section{Example}

In order to illustrate the second Noether Theorem for the multiple integral case, we will present the following example. Let $\mathbb{T}_{0}, \mathbb{T}_{1}, \mathbb{T}_{2}$, and $\mathbb{T}_{3}$ be time scales and let $\Omega \subseteq \mathbb{T}_{0} \times \mathbb{\mathbb { T }}_{1} \times \mathbb{T}_{2} \times \mathbb{\mathbb { T }}_{3}$ be an $\omega$-type set. For $i=0,1,2,3$, denote by $\sigma_{i}, \rho_{i}$, and $\Delta_{i}$ the forward jump operator, the backward jump operator, and the delta derivative on $\mathbb{T}_{i}$, respectively.

Let $t:=\left(t_{0}, t_{1}, t_{2}, t_{3}\right) \in \Omega$ and consider the following real functions defined on $\Omega: A_{0}, A_{1}, A_{2}, A_{3}$. Let $\mathbf{A}:=$ $\left(A_{1}, A_{2}, A_{3}\right)$ and denote

$$
\begin{aligned}
\nabla A_{0}(t):=\left(\frac{\partial A_{0}}{\Delta_{1} t_{1}}\left(\sigma_{0}\left(t_{0}\right), t_{1}, \sigma_{2}\left(t_{2}\right), \sigma_{3}\left(t_{3}\right)\right),\right. \\
\frac{\partial A_{0}}{\Delta_{2} t_{2}}\left(\sigma_{0}\left(t_{0}\right), \sigma_{1}\left(t_{1}\right), t_{2}, \sigma_{3}\left(t_{3}\right)\right), \\
\left.\frac{\partial A_{0}}{\Delta_{3} t_{3}}\left(\sigma_{0}\left(t_{0}\right), \sigma_{1}\left(t_{1}\right), \sigma_{2}\left(t_{2}\right), t_{3}\right)\right),
\end{aligned}
$$

$$
\begin{aligned}
\frac{\partial \mathbf{A}}{\Delta_{0} t_{0}}(t):=( & \frac{\partial A_{1}}{\Delta_{0} t_{0}}\left(t_{0}, \sigma_{1}\left(t_{1}\right), \sigma_{2}\left(t_{2}\right), \sigma_{3}\left(t_{3}\right)\right), \\
& \frac{\partial A_{2}}{\Delta_{0} t_{0}}\left(t_{0}, \sigma_{1}\left(t_{1}\right), \sigma_{2}\left(t_{2}\right), \sigma_{3}\left(t_{3}\right)\right), \\
& \left.\frac{\partial A_{3}}{\Delta_{0} t_{0}}\left(t_{0}, \sigma_{1}\left(t_{1}\right), \sigma_{2}\left(t_{2}\right), \sigma_{3}\left(t_{3}\right)\right)\right),
\end{aligned}
$$




$$
\begin{aligned}
\operatorname{curl} \mathbf{A}(t):=( & \frac{\partial A_{3}}{\Delta_{2} t_{2}}\left(\sigma_{0}\left(t_{0}\right), \sigma_{1}\left(t_{1}\right), t_{2}, \sigma_{3}\left(t_{3}\right)\right) \\
& -\frac{\partial A_{2}}{\Delta_{3} t_{3}}\left(\sigma_{0}\left(t_{0}\right), \sigma_{1}\left(t_{1}\right), \sigma_{2}\left(t_{2}\right), t_{3}\right), \\
& \frac{\partial A_{1}}{\Delta_{3} t_{3}}\left(\sigma_{0}\left(t_{0}\right), \sigma_{1}\left(t_{1}\right), \sigma_{2}\left(t_{2}\right), t_{3}\right) \\
& -\frac{\partial A_{3}}{\Delta_{1} t_{1}}\left(\sigma_{0}\left(t_{0}\right), t_{1}, \sigma_{2}\left(t_{2}\right), \sigma_{3}\left(t_{3}\right)\right), \\
& \frac{\partial A_{2}}{\Delta_{1} t_{1}}\left(\sigma_{0}\left(t_{0}\right), t_{1}, \sigma_{2}\left(t_{2}\right), \sigma_{3}\left(t_{3}\right)\right) \\
& \left.-\frac{\partial A_{1}}{\Delta_{2} t_{2}}\left(\sigma_{0}\left(t_{0}\right), \sigma_{1}\left(t_{1}\right), t_{2}, \sigma_{3}\left(t_{3}\right)\right)\right) .
\end{aligned}
$$

We will consider the following Lagrangian function:

$$
L=\frac{1}{2}\left\|\nabla A_{0}-\frac{\partial \mathbf{A}}{\Delta_{0} t_{0}}\right\|^{2}-\frac{1}{2}\|\operatorname{curl} \mathbf{A}\|^{2}
$$

that is the time scale version of the Lagrangian density for the electromagnetic field (see, e.g., [46]).

It can be proved that the functional

$$
\mathscr{L}=\int \cdots \int_{\Omega} L \Delta_{0} \Delta_{1} \Delta_{2} \Delta_{3}
$$

is invariant under the gauge transformations:

$$
\bar{A}_{k}=A_{k}+\frac{\partial}{\Delta_{k} t_{k}} p^{\rho_{k}}, \quad k=0,1,2,3,
$$

where $p: \Omega \rightarrow R$ is an arbitrary continuous function that has continuous partial delta derivatives of the first and second order (hence, we have equality of mixed partial delta derivatives; see [41]).

Since, for each $k=0,1,2,3$,

$$
T^{k}(p)=\frac{\partial}{\Delta_{k} t_{k}} p^{\rho_{k}},
$$

then, by Lemma 32, we conclude that

$$
\widetilde{T}^{k}(q)=-\frac{\partial}{\Delta_{k} t_{k}} q .
$$

Hence, from the second Noether theorem (Theorem 34), we get

$$
\sum_{k=0}^{3} \frac{\partial}{\Delta_{k} t_{k}} \widehat{E}_{k}(L) \equiv 0 \quad \text { on } \Omega^{\circ}
$$

where $\widehat{E}_{k}(L), k=0,1,2,3$ are the Euler-Lagrange expressions associated to functional $\mathscr{L}$.

If we suppose that, for each $k=0,1,2,3, A_{k}$ has continuous partial delta derivatives of the first and second order and that $A_{0}$ and the vector field $\mathbf{A}$ satisfy the so-called Lorentz conditions on time scales:

$$
\begin{aligned}
\left.\operatorname{div} \mathbf{A}\right|_{\left(t_{0}, \sigma_{1}\left(t_{1}\right), \sigma_{2}\left(t_{2}\right), \sigma_{3}\left(t_{3}\right)\right)} & =\left.\frac{\partial A_{0}}{\Delta_{0} t_{0}}\right|_{\left(t_{0}, \sigma_{1}\left(t_{1}\right), \sigma_{2}\left(t_{2}\right), \sigma_{3}\left(t_{3}\right)\right)} \\
\left.\operatorname{div} \mathbf{A}\right|_{\left(\sigma_{0}\left(t_{0}\right), t_{1}, \sigma_{2}\left(t_{2}\right), \sigma_{3}\left(t_{3}\right)\right)} & =\left.\frac{\partial A_{0}}{\Delta_{0} t_{0}}\right|_{\left(\sigma_{0}\left(t_{0}\right), t_{1}, \sigma_{2}\left(t_{2}\right), \sigma_{3}\left(t_{3}\right)\right)} \\
\left.\operatorname{div} \mathbf{A}\right|_{\left(\sigma_{0}\left(t_{0}\right), \sigma_{1}\left(t_{1}\right), t_{2}, \sigma_{3}\left(t_{3}\right)\right)} & =\left.\frac{\partial A_{0}}{\Delta_{0} t_{0}}\right|_{\left(\sigma_{0}\left(t_{0}\right), \sigma_{1}\left(t_{1}\right), t_{2}, \sigma_{3}\left(t_{3}\right)\right)}, \\
\left.\operatorname{div} \mathbf{A}\right|_{\left(\sigma_{0}\left(t_{0}\right), \sigma_{1}\left(t_{1}\right), \sigma_{2}\left(t_{2}\right), t_{3}\right)} & =\left.\frac{\partial A_{0}}{\Delta_{0} t_{0}}\right|_{\left(\sigma_{0}\left(t_{0}\right), \sigma_{1}\left(t_{1}\right), \sigma_{2}\left(t_{2}\right), t_{3}\right)},
\end{aligned}
$$

where $\operatorname{div} \mathbf{A}$ denotes the divergence of a vector field $\mathbf{A}$, that is,

$$
\operatorname{div} \mathbf{A}:=\frac{\partial A_{1}}{\Delta_{1} t_{1}}+\frac{\partial A_{2}}{\Delta_{2} t_{2}}+\frac{\partial A_{3}}{\Delta_{3} t_{3}},
$$

then the Euler-Lagrange expressions can be written in the following way:

$$
\begin{aligned}
\widehat{E}_{k}(L)= & \frac{\partial^{2} A_{k}}{\Delta_{0} t_{0}^{2}}\left(t_{0}, \sigma_{1}\left(t_{1}\right), \sigma_{2}\left(t_{2}\right), \sigma_{3}\left(t_{3}\right)\right) \\
& -\nabla^{2} A_{k}\left(t_{0}, t_{1}, t_{2}, t_{3}\right), \quad k=0,1,2,3,
\end{aligned}
$$

where

$$
\begin{aligned}
\nabla^{2} A_{k}(t):= & \frac{\partial^{2} A_{k}}{\Delta_{1} t_{1}^{2}}\left(\sigma_{0}\left(t_{0}\right), t_{1}, \sigma_{2}\left(t_{2}\right), \sigma_{3}\left(t_{3}\right)\right) \\
& +\frac{\partial^{2} A_{k}}{\Delta_{2} t_{2}^{2}}\left(\sigma_{0}\left(t_{0}\right), \sigma_{1}\left(t_{1}\right), t_{2}, \sigma_{3}\left(t_{3}\right)\right) \\
& +\frac{\partial^{2} A_{k}}{\Delta_{3} t_{3}^{2}}\left(\sigma_{0}\left(t_{0}\right), \sigma_{1}\left(t_{1}\right), \sigma_{2}\left(t_{2}\right), t_{3}\right) .
\end{aligned}
$$

Hence, under these assumptions, we can conclude that

$$
\begin{aligned}
\sum_{k=0}^{3} \frac{\partial}{\Delta_{k} t_{k}}\left(\frac{\partial^{2} A_{k}}{\Delta_{0} t_{0}^{2}}\left(t_{0}, \sigma_{1}\left(t_{1}\right), \sigma_{2}\left(t_{2}\right), \sigma_{3}\left(t_{3}\right)\right)\right. \\
\left.-\nabla^{2} A_{k}\left(t_{0}, t_{1}, t_{2}, t_{3}\right)\right)=0 \text { on } \Omega^{\circ} .
\end{aligned}
$$

\section{Concluding Remarks}

We proved that the important Noether's second theorem is valid not only for the continuous and discrete calculus but also for the quantum calculus. Moreover, in our opinion, the proofs presented in this paper are elegant and clear to follow.

The question of obtaining Noether's second theorem for multiple integrals with transformation of time in the time scale setting remains an interesting open question. To the best of the authors' knowledge, to extend the second Noether theorem to multiple integrals with transformation of time, substitution in the multiple integral is a fundamental tool and this result is not yet available in the literature. 
For other generalizations of the second Noether theorem, we refer the reader to [47] (in the context of the fractional calculus of variations) and [48] (in the context of the optimal control).

\section{Acknowledgments}

This work was supported by FEDER funds through COMPETE-Operational Programme Factors of Competitiveness ("Programa Operacional Factores de Competitividade") and by Portuguese funds through the Center for Research and Development in Mathematics and Applications (University of Aveiro) and the Portuguese Foundation for Science and Technology ("FCT-Fundação para a Ciência e a Tecnologia"), within Project PEst-C/MAT/UI4106/2011 with COMPETE no. FCOMP-01-0124-FEDER-022690. Agnieszka B. Malinowska was also supported by Bialystok University of Technology Grant S/WI/02/2011.

\section{References}

[1] E. Noether, "Invariante variationsprobleme," Nachrichten von der Gesellschaft der Wissenschaften zu Göttingen, pp. 235-257, 1918.

[2] Y. Kosmann-Schwarzbach, The Noether Theorems: Invariance and Conservation Laws in the Twentieth Century, Sources and Studies in the History of Mathematics and Physical Sciences, Springer, New York, NY, USA, 2011.

[3] T. M. Atanacković, S. Konjik, S. Pilipović, and S. Simić, "Variational problems with fractional derivatives: invariance conditions and Noether's theorem," Nonlinear Analysis: Theory, Methods \& Applications, vol. 71, no. 5-6, pp. 1504-1517, 2009.

[4] Z. Bartosiewicz and D. F. M. Torres, "Noether's theorem on time scales," Journal of Mathematical Analysis and Applications, vol. 342, no. 2, pp. 1220-1226, 2008.

[5] G. S. F. Frederico and D. F. M. Torres, "A formulation of Noether's theorem for fractional problems of the calculus of variations," Journal of Mathematical Analysis and Applications, vol. 334, no. 2, pp. 834-846, 2007.

[6] G. S. F. Frederico and D. F. M. Torres, "Noether's symmetry theorem for variational and optimal control problems with time delay," Numerical Algebra, Control and Optimization, vol. 2, no. 3, pp. 619-630, 2012.

[7] A. B. Malinowska, "A formulation of the fractional Noethertype theorem for multidimensional Lagrangians," Applied Mathematics Letters, vol. 25, no. 11, pp. 1941-1946, 2012.

[8] A. B. Malinowska and D. F. M. Torres, Introduction to the Fractional Calculus of Variations, Imperial College Press, London, UK, 2012.

[9] N. Martins and D. F. M. Torres, "Noether's symmetry theorem for nabla problems of the calculus of variations," Applied Mathematics Letters, vol. 23, no. 12, pp. 1432-1438, 2010.

[10] J. D. Logan, "On variational problems which admit an infinite continuous group," Yokohama Mathematical Journal, vol. 22, pp. 31-42, 1974.

[11] K. A. Aldwoah, A. B. Malinowska, and D. F. M. Torres, "The power quantum calculus and variational problems," Dynamics of Continuous, Discrete and Impulsive Systems B, vol. 19, no. 1-2, pp. 93-116, 2012.
[12] G. Bangerezako, "Variational q-calculus," Journal of Mathematical Analysis and Applications, vol. 289, no. 2, pp. 650-665, 2004.

[13] J. Baoguo, L. Erbe, and A. Peterson, "Oscillation of a family of $q$ difference equations," Applied Mathematics Letters, vol. 22, no. 6, pp. 871-875, 2009.

[14] A. M. C. Brito da Cruz, N. Martins, and D. F. M. Torres, "Higherorder Hahn's quantum variational calculus," Nonlinear Analysis: Theory, Methods \& Applications, vol. 75, no. 3, pp. 1147-1157, 2012.

[15] A. M. C. Brito da Cruz and N. Martins, "The $q$-symmetric variational calculus," Computers \& Mathematics with Applications, vol. 64, no. 7, pp. 2241-2250, 2012.

[16] A. M. C. Brito da Cruz, N. Martins, and D. F. M. Torres, "Hahn's symmetric quantum variational calculus," Numerical Algebra, Control and Optimization, vol. 3, no. 1, pp. 77-94, 2013.

[17] J. Cresson, G. S. F. Frederico, and D. F. M. Torres, "Constants of motion for non-differentiable quantum variational problems," Topological Methods in Nonlinear Analysis, vol. 33, no. 2, pp. 217231, 2009.

[18] A. B. Malinowska and N. Martins, "Generalized transversality conditions for the Hahn quantum variational calculus," Optimization, vol. 62, no. 3, pp. 323-344, 2013.

[19] A. B. Malinowska and D. F. M. Torres, "The Hahn quantum variational calculus," Journal of Optimization Theory and Applications, vol. 147, no. 3, pp. 419-442, 2010.

[20] N. Martins and D. F. M. Torres, "Higher-order infinite horizon variational problems in discrete quantum calculus," Computers \& Mathematics with Applications, vol. 64, no. 7, pp. 2166-2175, 2012.

[21] V. Kac and P. Cheung, Quantum Calculus, Springer, New York, NY, USA, 2002.

[22] S. Hilger, Ein maÿkettenkalkül mit anwendung auf zentrumsmannigfaltigkeiten [Ph.D. thesis], Universität Würzburg, Würzburg, Germany, 1988.

[23] M. Bohner, "Calculus of variations on time scales," Dynamic Systems and Applications, vol. 13, no. 3-4, pp. 339-349, 2004.

[24] R. Hilscher and V. Zeidan, "Calculus of variations on time scales: weak local piecewise $C_{r d}^{1}$ solutions with variable endpoints," Journal of Mathematical Analysis and Applications, vol. 289, no. 1, pp. 143-166, 2004.

[25] R. Almeida and D. F. M. Torres, "Isoperimetric problems on time scales with nabla derivatives," Journal of Vibration and Control, vol. 15, no. 6, pp. 951-958, 2009.

[26] M. J. Bohner, R. A. C. Ferreira, and D. F. M. Torres, "Integral inequalities and their applications to the calculus of variations on time scales," Mathematical Inequalities \& Applications, vol. 13, no. 3, pp. 511-522, 2010.

[27] R. A. C. Ferreira and D. F. M. Torres, "Higher-order calculus of variations on time scales," in Mathematical Control Theory and Finance, pp. 149-159, Springer, Berlin, Germany, 2008.

[28] R. A. C. Ferreira and D. F. M. Torres, "Isoperimetric problems of the calculus of variations on time scales," in Nonlinear Analysis and Optimization II. Optimization, vol. 514 of Contemporary Mathematics, pp. 123-131, American Mathematical Society, Providence, RI, USA, 2010.

[29] E. Girejko, A. B. Malinowska, and D. F. M. Torres, "The contingent epiderivative and the calculus of variations on time scales," Optimization, vol. 61, no. 3, pp. 251-264, 2012.

[30] A. B. Malinowska, N. Martins, and D. F. M. Torres, "Transversality conditions for infinite horizon variational problems on time scales," Optimization Letters, vol. 5, no. 1, pp. 41-53, 2011. 
[31] A. B. Malinowska and D. F. M. Torres, "Leitmann's direct method of optimization for absolute extrema of certain problems of the calculus of variations on time scales," Applied Mathematics and Computation, vol. 217, no. 3, pp. 1158-1162, 2010.

[32] A. B. Malinowska and D. F. M. Torres, "A general backwards calculus of variations via duality," Optimization Letters, vol. 5, no. 4, pp. 587-599, 2011.

[33] N. Martins and D. F. M. Torres, "Calculus of variations on time scales with nabla derivatives," Nonlinear Analysis: Theory, Methods \& Applications, vol. 71, no. 12, pp. e763-e773, 2009.

[34] N. Martins and D. F. M. Torres, "Generalizing the variational theory on time scales to include the delta indefinite integral," Computers \& Mathematics with Applications, vol. 61, no. 9, pp. 2424-2435, 2011.

[35] N. Martins and D. F. M. Torres, "Necessary optimality conditions for higher-order infinite horizon variational problems on time scales," Journal of Optimization Theory and Applications, vol. 155, no. 2, pp. 453-476, 2012.

[36] Z. Bartosiewicz, N. Martins, and D. F. M. Torres, "The second Euler-Lagrange equation of variational calculus on time scales," European Journal of Control, vol. 17, no. 1, pp. 9-18, 2011.

[37] M. Bohner and A. Peterson, Dynamic Equations on Time Scales, Birkhäauser, Boston, Mass, USA, 2001.

[38] M. Bohner and A. Peterso, Eds., Advances in Dynamic Equations on Time Scales, Birkhäauser, Boston, Mass, USA, 2003.

[39] P. E. Hydon and E. L. Mansfield, "Extensions of Noether's second theorem: from continuous to discrete systems," Proceedings of The Royal Society of London A, vol. 467, no. 2135, pp. 32063221, 2011.

[40] C. D. Ahlbrandt, M. Bohner, and J. Ridenhour, "Hamiltonian systems on time scales," Journal of Mathematical Analysis and Applications, vol. 250, no. 2, pp. 561-578, 2000.

[41] M. Bohner and G. Sh. Guseinov, "Partial differentiation on time scales," Dynamic Systems and Applications, vol. 13, no. 3-4, pp. 351-379, 2004.

[42] M. Z. Sarikaya, N. Aktan, H. Yıldırım, and K. İlarslan, "Partial $\Delta$-differentiation for multivariable functions on $n$-dimensional time scales," Journal of Mathematical Inequalities, vol. 3, no. 2, pp. 277-291, 2009.

[43] M. Bohner and G. Sh. Guseinov, "Multiple integration on time scales," Dynamic Systems and Applications, vol. 14, no. 3-4, pp. 579-606, 2005.

[44] M. Bohner and G. Sh. Guseinov, "Double integral calculus of variations on time scales," Computers \& Mathematics with Applications, vol. 54, no. 1, pp. 45-57, 2007.

[45] M. Bohner and G. Sh. Guseinov, "Line integrals and Green's formula on time scales," Journal of Mathematical Analysis and Applications, vol. 326, no. 2, pp. 1124-1141, 2007.

[46] J. D. Logan, Invariant Variational Principles, Academic Press, New York, NY, USA, 1977.

[47] A. B. Malinowska, "On fractional variational problems which admit local transformations," Journal of Vibration and Control, vol. 19, no. 8, pp. 1161-1169, 2013.

[48] D. F. M. Torres, "Gauge symmetries and Noether currents in optimal control," Applied Mathematics E-Notes, vol. 3, pp. 4957, 2003. 


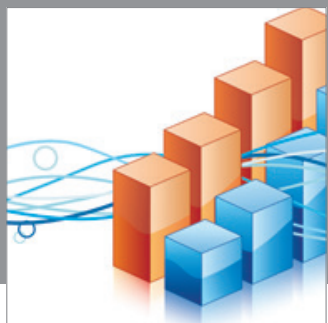

Advances in

Operations Research

mansans

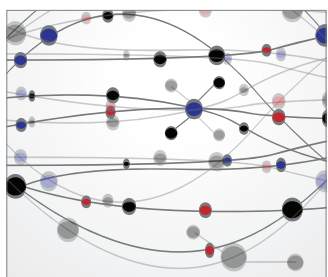

The Scientific World Journal
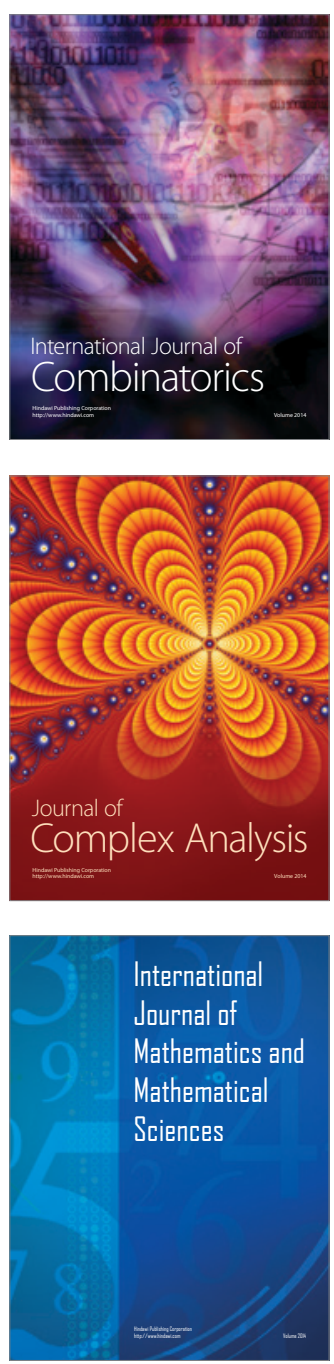
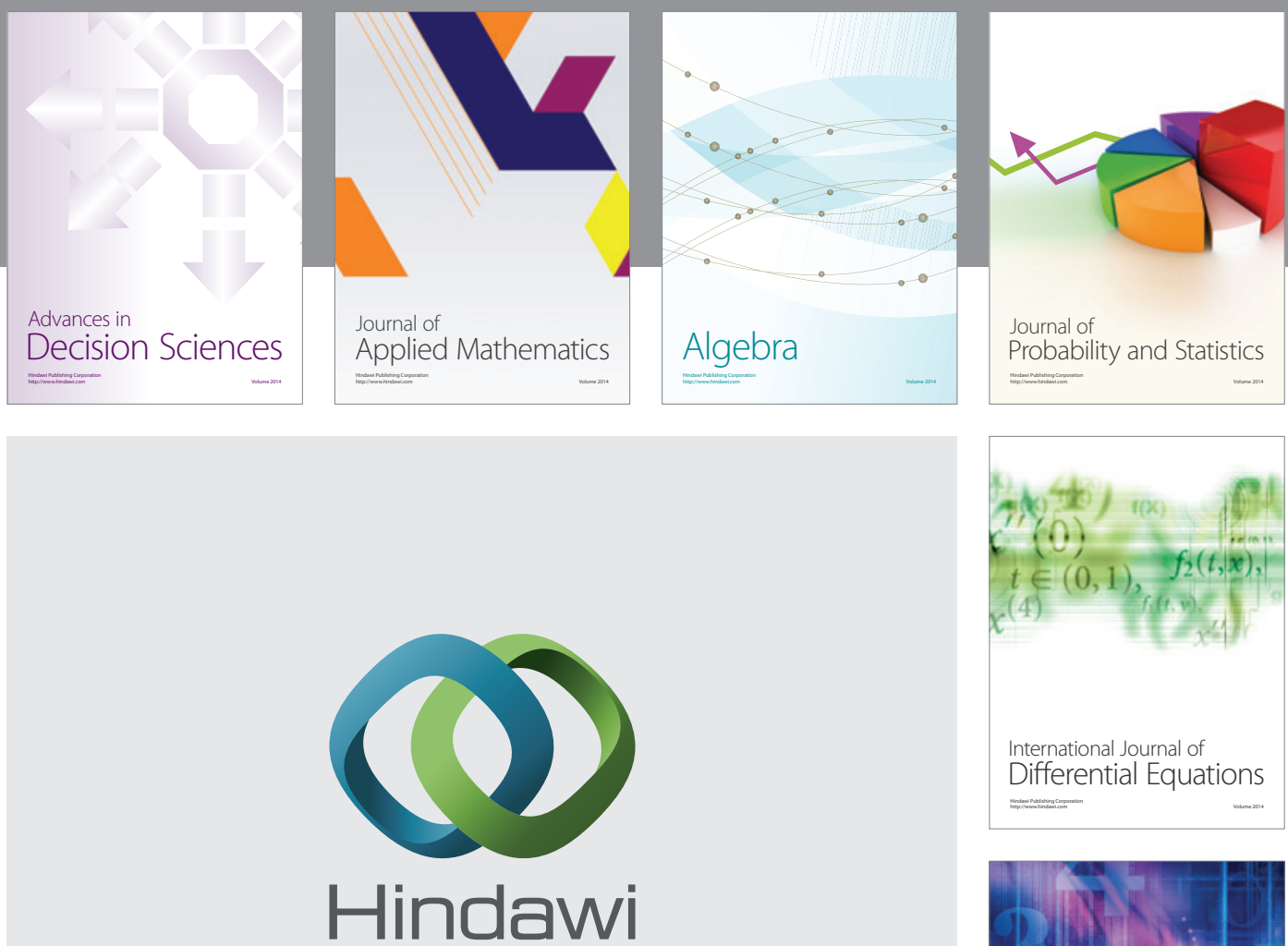

Submit your manuscripts at http://www.hindawi.com
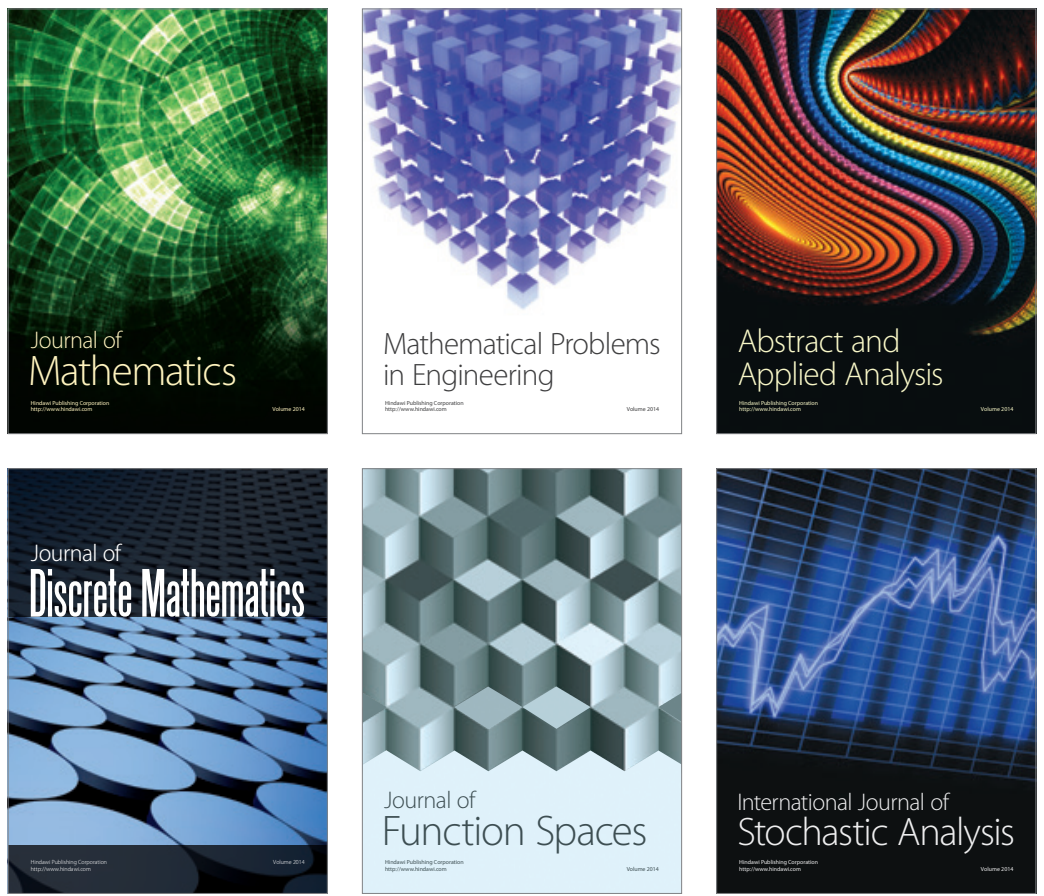

Journal of

Function Spaces

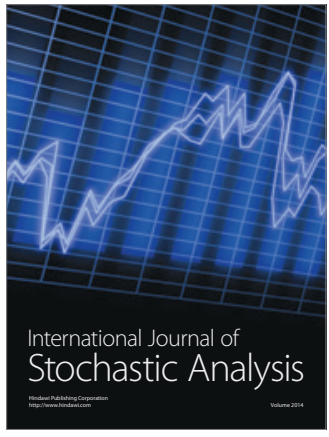

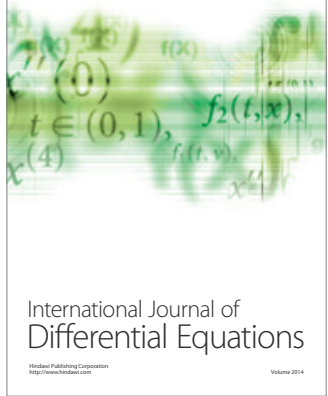
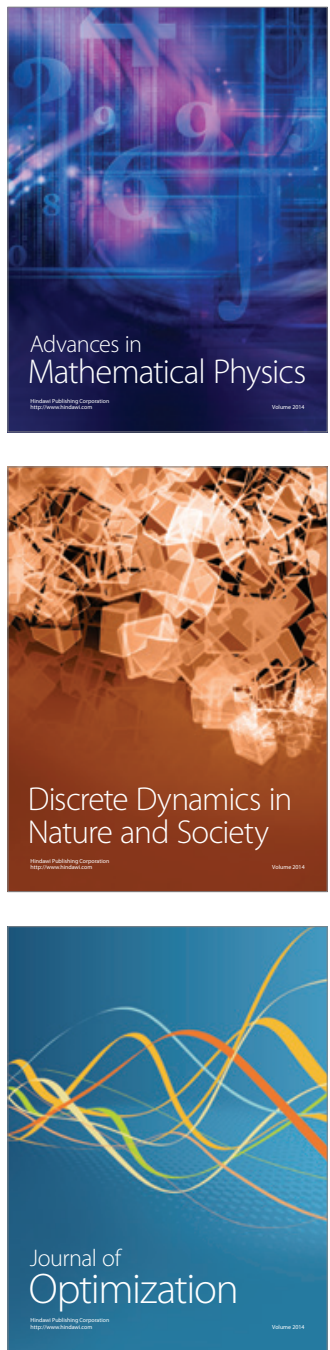